\title{
OPEN MAPPINGS OF THE UNIVERSAL CURVE ONTO CONTINUOUS CURVES
}

\author{
BY \\ DAVID C. WILSON( $\left.{ }^{1}\right)$
}

\begin{abstract}
A criterion for the existence of an open mapping from one compact metric space onto another is established in this paper. This criterion is then used to establish the existence of a monotone open mapping of the universal curve onto any continuous curve and the existence of a light open mapping of the universal curve onto any nondegenerate continuous curve. These examples show that if $f$ is a monotone open or a light open mapping of one compact space $X$ onto another $Y$, then it will not necessarily be the case that $\operatorname{dim} Y \leqq \operatorname{dim} X+k$, where $k$ is some positive integer.
\end{abstract}

1. Introduction. The two main theorems of this paper are the following:

THEOREM 1. There exists a monotone open map of the universal curve onto any continuous curve such that each point-inverse set is homeomorphic to the universal curve.

THEOREM 2. There exists a light open map of the universal curve onto any nondegenerate continuous curve such that each point-inverse set is a Cantor set.

R. D. Anderson announced Theorem 1 in 1956 [5]. However, since he never published a proof, the details are supplied here. In 1958 he conjectured [10] that there exists a light open map of the universal curve onto any $n$-cell. This question is answered by Theorem 2 .

The existence of open dimension raising mappings has been of interest for some time. The first light open dimension raising mapping was given by Kolmogoroff [16] in 1937. In this example the domain is a 1-dimensional continuous curve and the range is 2-dimensional. In 1954 Keldyš [15] constructed a similar example where the range is a 2-cell. In 1952 Anderson [6] constructed a monotone open map from a 1-dimensional continuum onto the Hilbert cube. The techniques of this paper are basic to the proofs of Theorems 1 and 2 .

Theorem 1 is of particular interest because of the following theorem of Dyer [13]: If $M$ and $N$ are compact metric spaces, $f$ is an open map of $M$ onto $N, f^{-1}(y)$ is a nondegenerate continuous curve for each $y \in Y$, and there exists $\varepsilon>0$ such that no simple closed curve in $M$ of diameter less than $\varepsilon$ is mapped to a point, then $\operatorname{dim} M$ $=\operatorname{dim} N+1$. A theorem of Alexandroff [1] states that if $X$ and $Y$ are continuous curves and $f$ is an open map of $X$ onto $Y$ such that each point-inverse set is countable, then

Presented to the Society, January 23, 1969; received by the editors April 2, 1971.

AMS 1970 subject classifications. Primary 54C10, 54F45; Secondary 54F50.

(1) This research was supported in part by the National Science Foundation, under NSF grant GP-7952X1. This paper contains most of the results in the author's Ph.D. thesis, written at Rutgers University under the supervision of Professor Louis F. McAuley. 
$\operatorname{dim} Y \leqq \operatorname{dim} X$. Theorem 2 shows that if a light open map has uncountable point-inverse sets, then the dimension of the range can be any positive integer or even infinite.

2. Open mappings. Let $(X, d)$ be any metric space. Let $G$ be any collection of subsets of $X$.

Notation. Let $G^{*}$ denote the subset of $X$ consisting of all points of $X$ which are in some member of $G$. If $A$ is a subset of $X$ and $\varepsilon$ a real number, then let $N_{\varepsilon}(A)$ $=\{x \in X$ : there exists $a \in A$ such that $d(x, a)<\varepsilon\}$. Let $d[A]$ denote the diameter of $A$. If $B$ is another subset of $X$, then let $d[A, B]$ denote the Hausdorff distance between $A$ and $B$. Let $\mu(G)=\max _{g \in G}\{d[g]\}$.

A compact metric space is called a compactum. A space is called a continuous curve if it is a connected and locally connected compactum. Int $(A)$ will denote the interior of $A$ relative to $X$.

Proposition 1. Let $X, Y, Z_{1}$, and $Z_{2}$ be compacta such that $X \subseteq Z_{1}$ and $Y \subseteq Z_{2}$. Suppose there exist two'sequences of finite collections of compacta, $F=\left\{F_{n}\right\}_{n=1}^{\infty}$ and $G=\left\{G_{n}\right\}_{n=1}^{\infty}$, with the following.properties:

1. $Z_{1} \supseteq F_{n}^{*} \supseteq F_{n+1}^{*} \supseteq X$ for all $n$ and $\bigcap_{n=1}^{\infty} F_{n}^{*}=X$.

2. $Z_{2} \supseteq G_{n}^{*} \supseteq G_{n+1}^{*} \supseteq Y$ for all $n$ and $\bigcap_{n=1}^{\infty} G_{n}^{*}=Y$.

3. Given $\varepsilon>0$ there exists $N$ such that $n>N$ implies $\mu\left(G_{n}\right)<\varepsilon$.

4. $T_{n}$ is a function of $F_{n}$ into $G_{n}$ such that

(a) if $f_{n} \in F_{n}, f_{n-1} \in F_{n-1}$, and $f_{n} \subseteq f_{n-1}$, then $T_{n}\left(f_{n}\right) \subseteq T_{n-1}\left(f_{n-1}\right)$,

(b) if $x \in X$, then there exists a nested sequence $\left\{f_{n}\right\}_{n=1}^{\infty}$ such that $x \in f_{n} \in F_{n}$.

5. If $f_{n}, f_{n}^{\prime} \in F_{n}$ and $f_{n} \cap f_{n}^{\prime} \neq \varnothing$, then $T_{n}\left(f_{n}\right) \cap T_{n}\left(f_{n}^{\prime}\right) \neq \varnothing$.

Then there exists a continuous function of $X$ into $Y$ defined by $g\left(\cap_{n=1}^{\infty} f_{n}\right)$ $=\bigcap_{n=1}^{\infty} T_{n}\left(f_{n}\right)$, where $f_{n}^{\prime} \in F_{n}$ and the sequence $\left\{f_{n}\right\}_{n=1}^{\infty}$ is nested.

Proof. We leave the proof to the reader.

Definition. A continuous function from $X$ onto $Y$ is called open if and only if the imáge of every open subset of $X$ is open in $Y$.

The next theorem is a generalization of Theorem 1 in [6].

Proposition 2. Let $X, Y$, and $Z$ be compacta such that $X \subseteq Z$. Suppose there exist two sequences of finite collections of compacta $F=\left\{F_{n}\right\}_{n=1}^{\infty}$ and $G=\left\{G_{n}\right\}_{n=1}^{\infty}$, with the following properties:

1. $G_{n}^{*}=Y$ for all $n$.

2. $Z \supseteq F_{n-1}^{*} \supseteq F_{n}^{*}$ for all $n$ and $\bigcap_{n=1}^{\infty} F_{n}^{*}=X$.

3. Given $\varepsilon>0$ there exists integer $n_{1}$ such that $n>n_{1}$ implies $\mu\left(G_{n}\right)<\varepsilon$.

4. There exists a one-to-one and onto correspondence between $F_{n}$ and $G_{n}$ given by $T_{n}$ such that

(a) if $f_{n} \in F_{n}, f_{n-1} \in F_{n-1}$, and $f_{n} \subseteq f_{n-1}$, then $T_{n}\left(f_{n}\right) \subseteq T_{n-1}\left(f_{n-1}\right)$,

(b) if $x \in X$, then there exists a nested sequence $\left\{f_{n}\right\}_{n=1}^{\infty}$ such that $x \in f_{n} \in F_{n}$,

(c) if $y \in Y$, then there exists a nested sequence $\left\{g_{n}\right\}_{n=1}^{\infty}$ such that $y \in g_{n} \in G_{n}$ and $T_{n}^{-1}\left(g_{n}\right) \subseteq T_{n-1}^{-1}\left(g_{n-1}\right)$.

5. If $f_{m a} f_{n}^{\prime} \in F_{n}$, then $f_{n} \cap f_{n}^{\prime} \neq \varnothing$ if and only if $T_{n}\left(f_{n}\right) \cap T_{n}\left(f_{n}^{\prime}\right) \neq \varnothing$. 
6. There exists $\eta>0$ such that if $f_{n}, f_{n}^{\prime} \in F_{n}$ and $f_{n} \cap f_{n}^{\prime} \neq \varnothing$, then $f_{n} \subseteq N_{n / 2^{n}}\left(f_{n}^{\prime}\right)$.

7. There exists $\mu>0$ such that if $f_{n} \in F_{n}, f_{n-1} \in F_{n-1}$, and $f_{n} \subseteq f_{n-1}$, then $f_{n-1}$ $\subseteq N_{\mu / 2^{n-1}}\left(f_{n}\right)$.

Then there exists an open mapping of $X$ onto $Y$ defined by

$$
g\left(\bigcap_{n=1}^{\infty} f_{n}\right)=\bigcap_{n=1}^{\infty} T_{n}\left(f_{n}\right)
$$

where $f_{n} \in F_{n}$ and the sequence $\left\{f_{n}\right\}_{n=1}^{\infty}$ is nested. Moreover, $g^{-1}\left(g\left(\bigcap_{n=1}^{\infty} f_{n}\right)\right)$ $=\bigcap_{n=1}^{\infty} f_{n}$.

Proof. By Proposition 1 , we know that $g$ is a continuous map of $X$ into $Y$. It is easy to show that the map $g$ is onto.

If $\left\{f_{n}\right\}_{n=1}^{\infty}$ and $\left\{f_{n}^{\prime}\right\}_{n=1}^{\infty}$ are nested sequences which have the property that there exists $f_{m} \in F_{m}$ such that $f_{n} \cap f_{m} \neq \varnothing$ and $f_{n}^{\prime} \cap f_{m} \neq \varnothing$, then Properties 5,6 , and 7 can be combined to show that $\bigcap_{n=1}^{\infty} f_{n}^{\prime} \subseteq N_{(1 / 2)^{m}(2 n+3 \mu)}\left(\bigcap_{n=1}^{\infty} f_{n}\right)$.

The map $g$ is open. Let $x \in X$ and let $V$ be any open subset of $X$ containing $x$. We must show that $g(x)$ is interior to $g(V)$. Choose $m$ large enough that $N_{(1 / 2)^{m}(2 \eta+3 \mu)}(x) \subseteq V$. Let $g_{m}^{1}, \ldots, g_{m}^{r}$ be all the members of $G_{m}$ which contain $g(x)$. Let $\left\{f_{n}\right\}_{n=1}^{\infty}$ be a nested sequence such that $x \in f_{n} \in F_{n}$ for all $n$. Let $y \in g_{m}^{i}$, where $1 \leqq i \leqq r$. Let $\left\{f_{n}^{\prime}\right\}_{n=1}^{\infty}$ be a nested sequence such that $f_{n} \in F_{n}$ for all $n$ and $g\left(\bigcap_{n=1}^{\infty} f_{n}^{\prime}\right)=y$. Since $y \in g_{m}^{i} \cap T_{m}\left(f_{m}^{\prime}\right), T_{m}^{-1}\left(g_{m}^{i}\right) \cap f_{m}^{\prime} \neq \varnothing$. Since $g(x) \in g_{m}^{i} \cap T_{m}\left(f_{m}\right)$ $T_{m}^{-1}\left(g_{m}^{i}\right) \cap f_{m} \neq \varnothing$. Therefore, $x \in \bigcap_{n=1}^{\infty} f_{n} \subseteq N_{(1 / 2)^{m}(2 n+3 \mu)}\left(\bigcap_{n=1}^{\infty} f_{n}^{\prime}\right)$, and there must exist $x^{\prime} \in \bigcap_{n=1}^{\infty} f_{n}^{\prime}$ such that $d\left(x, x^{\prime}\right)<\left(\frac{1}{2}\right)^{m}(2 \eta+3 \mu)$. Thus, $x^{\prime} \in V$. Since $g\left(x^{\prime}\right)=y$, by the definition of $g$, we have shown that $g(V)$ contains $g_{m}^{1} \cup \cdots \cup g_{m}^{r}$. Since $g(x) \in \operatorname{Int}\left(g_{m}^{1} \cup \cdots \cup g_{m}^{r}\right), g$ is an open mapping.

We now want to show that if $\left\{f_{n}\right\}_{n=1}^{\infty}$ is a nested sequence such that $f_{n} \in F_{n}$, then $g^{-1}\left(g\left(\bigcap_{n=1}^{\infty} f_{n}\right)\right)=\bigcap_{n=1}^{\infty} f_{n}$.

By the definition of $g$, we know that $g^{-1}\left(g\left(\cap_{n=1}^{\infty} f_{n}\right)\right)$ contains $\bigcap_{n=1}^{\infty} f_{n}$. If $g^{-1}\left(g\left(\bigcap_{n=1}^{\infty} f_{n}\right)\right) \neq \bigcap_{n=1}^{\infty} f_{n}$, then there exists $x^{\prime} \in g^{-1}\left(g\left(\bigcap_{n=1}^{\infty} f_{n}\right)\right)-\bigcap_{n=1}^{\infty} f_{n}$. Let $\left\{f_{n}^{\prime}\right\}_{n=1}^{\infty}$ be a nested sequence such that $x^{\prime} \in f_{n}^{\prime} \in F_{n}$ for all $n$. Choose $m$ large enough that $\left(\frac{1}{2}\right)^{m}(2 \eta+3 \mu)<\frac{1}{2} d\left[x^{\prime}, \bigcap_{n=1}^{\infty} f_{n}\right]$. Since $g\left(x^{\prime}\right)=g\left(\bigcap_{n=1}^{\infty} f_{n}\right), T_{m}\left(f_{m}\right) \cap T_{m}\left(f_{m}^{\prime}\right) \neq \varnothing$. Therefore, $f_{m} \cap f_{m}^{\prime} \neq \varnothing$ so that $\bigcap_{n=1}^{\infty} f_{n}^{\prime} \subseteq N_{(1 / 2)^{m}(2 n+3 \mu)}\left(\bigcap_{n=1}^{\infty} f_{n}\right)$. Since $x^{\prime} \in \bigcap_{n=1}^{\infty} f_{n}^{\prime}$, there exists $x \in \bigcap_{n=1}^{\infty} f_{n}$ such that $d\left(x, x^{\prime}\right)<\left(\frac{1}{2}\right)^{m}(2 \eta+3 \mu)$. Therefore, $d\left(x^{\prime}, \bigcap_{n=1}^{\infty} f_{n}\right)$ $\leqq d\left(x, x^{\prime}\right)<\frac{1}{2} d\left[x^{\prime}, \bigcap_{n=1}^{\infty} f_{n}\right]$, a contradiction. Thus, $g^{-1}\left(g\left(\bigcap_{n=1}^{\infty} f_{n}\right)\right)=\bigcap_{n=1}^{\infty} f_{n}$, and we have established our proposition.

Notation. If $G$ is a collection of subsets of $X$ and $A$ is a subset of $X$, then let St $(A, G)=\{g \in G: g \cap A \neq \varnothing\}$. This collection is called the star of $A$. Inductively, let $\mathrm{St}^{n}(A, G)=\operatorname{St}\left(\mathrm{St}^{n-1}(A, G)^{*}, G\right)$. For convenience let $\mathrm{St}^{0}(A, G)$ denote the collection of all members of $G$ contained in $A$. Let $\operatorname{St}^{k}(A, G)$ be the empty collection for all negative integers $k$.

When R. D. Anderson proved Theorem 1 in [6], he added additional inductive conditions to the original hypotheses in order to prove the theorem. For our purposes it is convenient to isolate these conditions into a separate proposition. 
Proposition 3. Let $X, Y$, and $Z$ be compacta such that $X \subseteq Z$. Suppose there exist two sequences $J=\left\{J_{n}\right\}_{n=1}^{\infty}$ and $K=\left\{K_{n}\right\}_{n=1}^{\infty}$ of finite collections of compacta with the following properties:

1. $J_{1}=\{Y\}$ and $J_{n}^{*}=Y$ for all $n$.

2. $K_{1}=\{Z\}, K_{n-1}^{*} \supseteq K_{n}^{*}$, and $\bigcap_{n=1}^{\infty} K_{n}^{*}=X$.

3. Given $\varepsilon>0$ there exists $m$ such that $n>m$ implies $\mu\left(J_{n}\right)<\varepsilon$.

4. The members of $J_{n}$ and $K_{n}$ have disjoint nonempty interiors.

5. There exists an integer $L_{n}>1$ with the property that if $j_{n} \in J_{n}$ and $j_{n-1}^{1}, \ldots, j_{n-1}^{r}$ are all the members of $J_{n-1}$ which meet $\operatorname{St}^{L_{n}+1}\left(j_{n}, J_{n}\right)^{*}$, then $j_{n-1}^{1} \cap \cdots \cap j_{n-1}^{r} \neq \varnothing$.

6. There exists a one-to-one and onto correspondence between $J_{n}$ and $K_{n}$ given by $R_{n}$ such that $R_{n}\left(j_{n}\right) \cap R_{n}\left(j_{n}^{\prime}\right) \neq \varnothing$ if and only if $j_{n} \cap j_{n}^{\prime} \neq \varnothing$. There exists $\eta>0$ such that if $j_{n} \cap j_{n}^{\prime} \neq \varnothing$, then $R_{n}\left(j_{n}\right) \subseteq N_{n / 2^{n-1}}\left(R_{n}\left(j_{n}^{\prime}\right)\right)$.

7. If $j_{n} \in J_{n}$ and $j_{n-1} \in J_{n-1}$, then $R_{n}\left(j_{n}\right) \cap R_{n-1}\left(j_{n-1}\right) \neq \varnothing$ if and only if $j_{n-1}$ meets $\operatorname{St}^{L_{n}}\left(j_{n}, J_{n}\right)^{*}$. Also if $k_{n} \in K_{n}$ and $k_{n-1} \in K_{n-1}$ and $k_{n} \cap k_{n-1} \neq \varnothing$, then $k_{n}$ $\cap$ Int $\left(k_{n-1}\right) \neq \varnothing$.

8. There exists $\lambda>0$, such that if $j_{n} \cap j_{n-1} \neq \varnothing$, then $R_{n-1}\left(j_{n-1}\right) \subseteq N_{\lambda / 2^{n}}\left(R_{n}\left(j_{n}\right)\right)$.

Then there exists an open map $g$ of $X$ onto $Y$, which has the property that $g^{-1}\left(g\left(\bigcap_{n=1}^{\infty} f_{n}\right)\right)=\bigcap_{n=1}^{\infty} f_{n}$, where $\left\{f_{n}\right\}_{n=1}^{\infty}$ is a nested sequence such that $f_{n}=R_{n}\left(j_{n}^{1}\right)$ $\cup \cdots \cup R_{n}\left(j_{n}^{r}\right)$, where $j_{n}^{1} \cup \cdots \cup j_{n}^{r} \in G_{n}$. $\left(G_{n}\right.$ is defined below. $)$

Proof. Let $G_{n}^{\prime}=\left\{j_{n}^{1} \cup \cdots \cup j_{n}^{r}: j_{n}^{1} \cap \cdots \cap j_{n}^{r} \neq \varnothing, j_{n}^{i} \in J_{n}\right\}$. A member $g$ of $G_{n}^{\prime}$ is in $G_{n}$ if and only if $g=j_{n}^{1} \cup \cdots \cup j_{n}^{r}$ and $j_{n}^{1} \cap \cdots \cap j_{n}^{r} \cap j=\varnothing$ for all $j \notin\left\{j_{n}^{1}, \ldots, j_{n}^{r}\right\}$. Note that $G_{n}^{*}=Y$.

Since the members of $J_{n}$ have nonempty disjoint interiors, each member of $G_{n}$ can be written uniquely as a union of members of $J_{n}$. If $g_{n}=j_{n}^{1} \cup \cdots \cup j_{n}^{r}$, then define $T_{n}^{-1}\left(g_{n}\right)=R_{n}\left(j_{n}^{1}\right) \cup \cdots \cup R_{n}\left(j_{n}^{r}\right)$. Since $g_{n}$ is written uniquely as a union of members of $J_{n}, T_{n}^{-1}$ is a well-defined function from $G_{n}$ onto the collection $F_{n}$ $=\left\{T_{n}^{-1}\left(g_{n}\right): g_{n} \in G_{n}\right\}$. Since distinct members of $K_{n}$ have disjoint nonempty interiors, $T_{n}^{-1}$ is one-to-one. Therefore, $T_{n}$ is well defined.

The proof of Proposition 3 will be a verification of the seven properties listed in the hypotheses of Proposition 2. Since most of this checking is routine, only a few properties are verified here.

Property 4b. Let $x \in X$. We must find a nested sequence $\left\{f_{n}\right\}_{n=1}^{\infty}$ such that $x \in f_{n} \in F_{n}$ for all $n$.

Let $f_{1}=Z$. For $n>2$ choose $k_{n} \in K_{n}$ such that $x \in k_{n}$. Let $k_{n-1}^{1}, \ldots, k_{n-1}^{r}$ be all the members of $K_{n-1}$ which have the property that $R_{n-1}^{-1}\left(k_{n-1}^{i}\right)$ meets $\mathrm{St}^{L_{n}+1}\left(R_{n}^{-1}\left(k_{n}\right), J_{n}\right)^{*}$. If $k_{n-1}$ is a member of $K_{n-1}$ which contains $x$, then $x \in k_{n}$ $\cap k_{n-1}$, so that by hypothesis $7, R_{n-1}^{-1}\left(k_{n-1}\right)$ meets $\operatorname{St}^{L_{n}}\left(R_{n}^{-1}\left(k_{n}\right), J_{n}\right)^{*}$. Therefore, $k_{n-1} \in\left\{k_{n-1}^{1}, \ldots, k_{n-1}^{r}\right\}$ and $x \in k_{n-1}^{1} \cup \cdots \cup k_{n-1}^{r}$.

Now choose $f_{n-1}$ to be any member of $F_{n-1}$ which contains $k_{n-1}^{1} \cup \cdots \cup k_{n-1}^{r}$. (There does exist such a member of $F_{n-1}$, because by hypothesis 5 , we know that $R_{n-1}^{-1}\left(k_{n-1}^{1}\right) \cap \cdots \cap R_{n-1}^{-1}\left(k_{n-1}^{r}\right) \neq \varnothing$.) We want to show that if $f_{n-1}$ has been chosen 
as above for all $n$, then the sequence $\left\{f_{n-1}\right\}_{n=2}^{\infty}$ is nested. Since $x \in k_{n}$, we know that $k_{n}$ will be contained in $f_{n}$. Thus, $f_{n} \subseteq \mathrm{St}\left(k_{n}, K_{n}\right)^{*}$. Therefore, it is sufficient to show that St $\left(k_{n}, K_{n}\right)^{*} \subseteq k_{n-1}^{1} \cup \cdots \cup k_{n-1}^{r}$. If $k_{n}^{\prime} \in \operatorname{St}\left(k_{n}, K_{n}\right)$ and $k_{n}^{\prime} \cap k_{n-1} \neq \varnothing$, where $k_{n-1} \in K_{n-1}$, then $R_{n-1}^{-1}\left(k_{n-1}\right)$ meets $\operatorname{St}^{L_{n}}\left(R_{n}^{-1}\left(k_{n}^{\prime}\right), J_{n}\right)^{*}$. Since $k_{n} \cap k_{n}^{\prime} \neq \varnothing$, $R_{n}^{-1}\left(k_{n}\right) \cap R_{n}^{-1}\left(k_{n}^{\prime}\right) \neq \varnothing$, and thus $R_{n-1}^{-1}\left(k_{n-1}\right)$ meets $\operatorname{St}^{L_{n}+1}\left(R_{n}^{-1}\left(k_{n}\right), J_{n}\right)^{*}$. Therefore, $k_{n-1}$ is a member of $\left\{k_{n-1}^{1}, \ldots, k_{n-1}^{r}\right\}$. Therefore, $k_{n}^{\prime} \subseteq k_{n-1}^{1} \cup \cdots \cup k_{n-1}^{r}$.

Property 4c. Let $y \in Y$. Choose $j_{n} \in J_{n}$ such that $y \in j_{n}$. Let $j_{n-1}^{1}, \ldots, j_{n-1}^{r}$ be all the members of $J_{n-1}$ which meet $\operatorname{St}_{n}^{L_{n}+1}\left(j_{n}, J_{n}\right)^{*}$. Since $j_{n-1}^{1} \cap \cdots \cap j_{n-1}^{r} \neq \varnothing$, there exists $g_{n-1} \in G_{n-1}$ which contains $j_{n-1}^{1} \cup \cdots \cup j_{n-1}^{r}$. We want to show that the sequence $\left\{g_{n-1}\right\}_{n=2}^{\infty}$ has the required properties.

Note that $y \in g_{n-1}$. For if $y \in j_{n-1}$, then $j_{n} \cap j_{n-1} \neq \varnothing$, and $j_{n-1}$ meets St $\left(j_{n}, J_{n}\right)^{*}$. Therefore, $j_{n-1}$ meets $\mathrm{St}^{L_{n}+1}\left(j_{n}, J_{n}\right)^{*}$ and $j_{n-1} \in\left\{j_{n-1}^{1}, \ldots, j_{n-1}^{r}\right\}$. Thus, $y \in j_{n-1}^{1}$ $\cup \cdots \cup j_{n-1}^{r} \subseteq g_{n-1}$.

We must show that $g_{n} \subseteq g_{n-1}$. Since $y \in j_{n}, j_{n} \subseteq g_{n}$. Therefore, it is sufficient to show that St $\left(j_{n}, J_{n}\right)^{*} \subseteq j_{n-1}^{1} \cup \cdots \cup j_{n-1}^{r}$. If $j_{n}^{\prime} \in \operatorname{St}\left(j_{n}, J_{n}\right)$ and $j_{n}^{\prime} \cap j_{n-1} \neq \varnothing$, where $j_{n-1} \in J_{n-1}$, then $j_{n-1} \cap \operatorname{St}\left(j_{n}^{\prime}, J_{n}\right)^{*} \neq \varnothing$. Hence, $j_{n-1} \cap \operatorname{St}^{2}\left(j_{n}, J_{n}\right)^{*} \neq \varnothing \quad$ and $j_{n-1} \in\left\{j_{n-1}^{1}, \ldots, j_{n-1}^{r}\right\}$. Thus, St $\left(j_{n}, J_{n}\right)^{*} \subseteq j_{n-1}^{1} \cup \cdots \cup j_{n-1}^{r}$.

We want to prove that $T_{n}^{-1}\left(g_{n}\right) \subseteq T_{n-1}^{-1}\left(g_{n-1}\right)$. It is sufficient to show that if $j_{n}^{\prime} \in \operatorname{St}\left(j_{n}, J_{n}\right)$, then $R_{n}\left(j_{n}^{\prime}\right) \subseteq R_{n-1}\left(j_{n-1}^{1}\right) \cup \cdots \cup R_{n-1}\left(j_{n-1}^{r}\right)$. If $j_{n}^{\prime} \in \operatorname{St}\left(j_{n}, J_{n}\right)$ and $R_{n}\left(j_{n}^{\prime}\right) \cap R_{n-1}\left(j_{n-1}\right) \neq \varnothing$, then $j_{n-1} \cap \mathrm{St}^{L_{n}}\left(j_{n}^{\prime}, J_{n}\right)^{*} \neq \varnothing$. Thus, $j_{n-1} \cap \mathrm{St}^{L_{n}+1}\left(j_{n}, J_{n}\right)^{*}$ $\neq \varnothing$ and $j_{n-1} \in\left\{j_{n-1}^{1}, \ldots, j_{n-1}^{r}\right\}$. Therefore, $R_{n}\left(j_{n}^{\prime}\right) \subseteq R_{n-1}\left(j_{n-1}^{1}\right) \cup \cdots \cup R_{n-1}\left(j_{n-1}^{r}\right)$.

Property 6. Let $f_{n}, f_{n}^{\prime} \in F_{n}$ such that $f_{n} \cap f_{n}^{\prime} \neq \varnothing$. Thus, there exist $k_{n}, k_{n}^{\prime} \in K_{n}$ such that $k_{n} \subseteq f_{n}, k_{n}^{\prime} \subseteq f_{n}^{\prime}$, and $k_{n} \cap k_{n}^{\prime} \neq \varnothing$. By hypothesis 6 , we have

$$
f_{n} \subseteq N_{\eta / 2^{n-1}}\left(k_{n}\right) \subseteq N_{n / 2^{n-1}}\left(N_{\eta / 2^{n-1}}\left(k_{n}^{\prime}\right)\right) \subseteq N_{n / 2^{n}}\left(k_{n}^{\prime}\right) \subseteq N_{n / 2^{n}}\left(f_{n}^{\prime}\right) .
$$

Property 7. Let $f_{n} \in F_{n}$ and $f_{n-1} \in F_{n-1}$ be chosen such that $f_{n} \subseteq f_{n-1}$. If $j_{n} \in J_{n}$ and $j_{n} \subseteq T_{n}\left(f_{n}\right)$, then since $T_{n}\left(f_{n}\right) \subseteq T_{n-1}\left(f_{n-1}\right)$, there exists $j_{n-1} \subseteq T_{n-1}\left(f_{n-1}\right)$ such that $j_{n} \cap j_{n-1} \neq \varnothing$. Since $R_{n-1}\left(j_{n-1}\right) \subseteq N_{\lambda / 2^{n}}\left(R_{n}\left(j_{n}\right)\right), \quad f_{n-1} \subseteq N_{n / 2^{n-1}}\left(R_{n-1}\left(j_{n-1}\right)\right)$ $\subseteq N_{\eta / 2^{n-1}}\left(N_{\lambda / 2^{n}}\left(R_{n}\left(j_{n}\right)\right)\right) \subseteq N_{(1 / 2)^{n}(2 n+\lambda)}\left(R_{n}\left(j_{n}\right)\right) \subseteq N_{(1 / 2)^{n}(2 \eta+\lambda)}\left(f_{n}\right)$. If we let

we have Property 7.

$$
\mu=\frac{1}{2}(2 \eta+\lambda)
$$

Proposition 3 now follows from Proposition 2.

\section{The two main theorems.}

Definition of THE UNIVERSAL CURVE. Let $N$ be the set of points in $E^{3}$ for which $0 \leqq x \leqq 1,0 \leqq y \leqq 1$, and $0 \leqq z \leqq 1$. For $w=x, y, z$ and $i=1,2,3, \ldots$, let $D_{i}(w)$ be the collection of all open intervals on the $w$-axis of length $1 / 3^{i}$ whose endpoints have $w$-coordinates which are positive rational numbers less than 1 , the expression for each such rational number having $3^{i}$ as a denominator when in lowest terms. Let $M$ be the set of all points $(x, y, z)$ of $N$ for which for no $i$ do two of the points $(x, 0,0),(0, y, 0)$, and $(0,0, z)$ belong to the set $D_{i}^{*}(x) \cup D_{i}^{*}(y) \cup D_{i}^{*}(z)$. The set $M$ is called the universal curve. 
Notation. If $A \subseteq X$, then $\mathrm{Bd}(A)$ will denote the boundary of $A$ relative to $X$. $\mathrm{Cl}(A)$ will denote the closure of $A$ relative to $X$. If $G$ is a collection of subsets of $X$, then let $G(A)$ denote the collection of all members of $G$ which are contained in $A$. Let $G^{\prime}(A)$ denote the collection of elements of $G$ not contained in $A$. Let $Z_{G}(A)$ be those elements of $G(A)$ which meet elements of $G^{\prime}(A)$. Let $|G|$ denote the cardinality of $G$.

DeFINITION. If $G$ is a collection of point sets, then $G$ is said to be simple provided

1. For each $g \in G, g-\mathrm{Bd}(g)$ is connected and $\mathrm{Cl}(g-\mathrm{Bd}(g))=g$.

2. Distinct members of $G$ have disjoint interiors relative to $G^{*}$.

Definition. A finite collection of sets $G=\left\{g_{1}, \ldots, g_{s}\right\}$ is called a simple chain if $g_{i} \cap g_{k} \neq \varnothing$ if and only if $|i-k| \leqq 1$.

Note. In this paper it will always be the case that simple chains are simple collections.

The terms interlace, 1-dimensional collection, $A$-defining sequence, and $B$ defining sequence are all defined in [2] so that we will not define them here. In the same paper R. D. Anderson showed that every 1-dimensional continuum for which there exists a $B$-defining sequence is homeomorphic to the universal curve.

Proposition 4. If $Y$ is any continuous curve, then there exist two sequences of finite collections of continua $J=\left\{J_{n}\right\}_{n=1}^{\infty}$ and $K=\left\{K_{n}\right\}_{n=1}^{\infty}$ with the following properties:

1. $J_{1}=\{Y\}$ and $J_{n}^{*}=Y$ for all $n$.

2. $K_{1}=\left\{I^{3}\right\}$ and $K_{n}^{*} \subseteq K_{n-1}^{*}$ for all $n$.

3. $\mu\left(J_{n}\right)<1 / n$.

4. $J_{n}$ and $K_{n}$ are simple collections.

5. There exists an integer $L_{n}>1$ with the property that if $j_{n} \in J_{n}$ and $j_{n-1}^{1}, \ldots, j_{n-1}^{r}$ are all the members of $J_{n-1}$ which meet $\operatorname{St}^{L_{n}+1}\left(j_{n}, J_{n}\right)^{*}$, then $j_{n-1}^{1} \cap \cdots \cap j_{n-1}^{r} \neq \varnothing$.

6. There exists a one-to-one and onto correspondence between $J_{n}$ and $K_{n}$ given by $R_{n}$ such that $R_{n}\left(j_{n}\right) \cap R_{n}\left(j_{n}^{\prime}\right) \neq \varnothing$ if and only if $j_{n} \cap j_{n}^{\prime} \neq \varnothing$. If $j_{n} \cap j_{n}^{\prime} \neq \varnothing$, then $R_{n}\left(j_{n}\right) \subseteq N_{8 / 2^{n-1}}^{\prime \prime}\left(R_{n}\left(j_{n}^{\prime}\right)\right)$.

7. If $j_{n} \in J_{n}$ and $j_{n-1} \in J_{n-1}$, then $R_{n}\left(j_{n}\right) \cap R_{n-1}\left(j_{n-1}\right) \neq \varnothing$ if and only if $j_{n-1}$ meets $\operatorname{St}^{L_{n}}\left(j_{n}, J_{n}\right)^{*}$. Also, if $k_{n} \in K_{n}, k_{n-1} \in K_{n-1}$, and $k_{n} \cap k_{n-1} \neq \varnothing$, then

$$
k_{n} \cap \operatorname{Int}\left(k_{n-1}\right) \neq \varnothing \text {. }
$$

8. If $j_{n} \in J_{n}, j_{n-1} \in J_{n-1}$, and $j_{n} \cap j_{n-1} \neq \varnothing$, then $R_{n}\left(j_{n}\right)$ meets every member of $H_{n-1}\left(R_{n-1}\left(j_{n-1}\right)\right)$ and $R_{n-1}\left(j_{n-1}\right) \subseteq N_{8 / 2^{n}}\left(R_{n}\left(j_{n}\right)\right)$.

9. There exists, a finite simple collection of polyhedral 3-cells $H_{n}$ such that $H_{n}$ refines $K_{n}, K_{n}^{*}=H_{n}^{*}$, and $H=\left\{H_{n}\right\}_{n=1}^{\infty}$ is a $B$-defining sequence. Also, $\mu\left(H_{n}\right)<4 / 2^{n}$. Distinct members of $H_{n}$ meet in the empty set or in a 2-cell.

10. For each $j_{n} \in J_{n}$ there exists a collection $A_{j_{n}} \subseteq H_{n}$ such that $A_{j_{n}}^{*} \subseteq R_{n}\left(j_{n}\right)$ and such that if $j_{n}^{\prime} \in J_{n}$ and $j_{n} \cap j_{n}^{\prime} \neq \varnothing$, then $R_{n}\left(j_{n}^{\prime}\right)$ meets each member of $A_{j_{n}}$ and each component of $R_{n}\left(j_{n}^{\prime}\right)$ meets some member of $A_{j_{n}}$. Each component of $R_{n}\left(j_{n}\right)$ will contain exactly one member of $A_{j_{n}}$. No member of $H_{n}$ will meet two members of the collection $\left\{a \in A_{j_{n}}: j_{n} \in J_{n}\right\}$. 
Proof. We can choose the metric on $Y$ so that $d[Y]<1$. Define $R_{1}$ between $J_{1}$ and $K_{1}$ by $R_{1}(Y)=I^{3}$. Since $d[Y]<1, \mu\left(J_{1}\right)<1$.

Let $H_{1}=\left\{I^{3}\right\}$. Since $d\left[I^{3}\right]=3^{1 / 2}<2, \mu\left(H_{1}\right)<4 / 2^{1}$. All of the other conditions in the first stage of the induction are trivially satisfied. Assume the theorem for the integer $n$.

We now want to define a function $\gamma_{n}$ from the collection $E=\left\{h \cap h^{\prime}: h, h^{\prime} \in H_{n}\right.$ and $\left.h \cap h^{\prime} \neq \varnothing\right\}$ into the subsets of $I^{3}$ with the following properties:

1. $\gamma_{n}\left(h \cap h^{\prime}\right)$ is a polyhedral 3-cell contained in Int $\left(h \cup h^{\prime}\right)$.

2. The members of the collection $\left\{\gamma_{n}\left(h \cap h^{\prime}\right): h \neq h^{\prime}\right\}$ are pairwise disjoint.

3. If $h$ and $h^{\prime}$ are distinct members of $H_{n}$ and $h \cap h^{\prime} \neq \varnothing$, then $\gamma_{n}\left(h \cap h^{\prime}\right) \cap \gamma_{n}(h)$ $=2$-cell and $\gamma_{n}\left(h \cap h^{\prime}\right) \cap h \cap h^{\prime}=2$-cell.

Moreover, we want to require that there exists a simple chain of polyhedral 3-cells, $\Gamma_{n}\left(h \cap h^{\prime}\right)$ such that.

1. The first member of $\Gamma_{n}\left(h \cap h^{\prime}\right)$ is $\gamma_{n}(h)$ and the last is $\gamma_{n}\left(h^{\prime}\right)$ (or vice versa).

2. $\Gamma_{n}\left(h \cap h^{\prime}\right)^{*}=\gamma_{n}(h) \cup \gamma_{n}\left(h \cap h^{\prime}\right) \cup \gamma_{n}\left(h^{\prime}\right)$.

3. $\Gamma_{n}\left(h \cap h^{\prime}\right)^{*}$ refines $\left\{h, h^{\prime}\right\}$ and has an equal number of members in each of $h$ and $h^{\prime}$.

4. Consecutive members of $\Gamma_{n}\left(h \cap h^{\prime}\right)$ meet in 2-cells.

5. $\mu\left(\Gamma_{n}\left(h \cap h^{\prime}\right)\right)<4\left(\frac{1}{2}\right)^{n+1}$ and if $h \neq h^{\prime}$, then $\left|\Gamma_{n}\left(h \cap h^{\prime}\right)\right|>50$.

Let $\gamma_{n}(h)$ be a polyhedral 3-cell in the interior of $h$ such that $d\left[\gamma_{n}(h)\right]<4\left(\frac{1}{2}\right)^{n+1}$. (The set $\gamma_{n}(h)$ can be taken to be a cube.) Let $\Gamma_{n}(h)=\left\{\gamma_{n}(h)\right\}$. Let $h \cap h^{\prime}$ be a member of $E$, where $h \neq h^{\prime}$. Let $A$ be a polygonal arc in the interior of $h \cup h^{\prime}$ such that $A$ meets the 2-cell $h \cap h^{\prime}$ in one point, and $A$ meets each of $\gamma_{n}(h)$ and $\gamma_{n}\left(h^{\prime}\right)$ in an endpoint of $A$. We can assume the members of the collection of all such arcs are pairwise disjoint with the same properties as the old. "Fatten" each arc slightly so that the fattened arcs remain disjoint and meet $\gamma_{n}(h)$ in a 2-cell. The set $\gamma_{n}\left(h \cap h^{\prime}\right)$ will denote the fattened arc between $\gamma_{n}(h)$ and $\gamma_{n}\left(h^{\prime}\right)$. It is now clear that we can find a simple chain of 3-cells $\Gamma_{n}\left(h \cap h^{\prime}\right)$ with the desired properties. Let $\Gamma_{n}$ denote the collection of all 3 -cells which are members of some $\Gamma_{n}\left(h \cap h^{\prime}\right)$.

Let $y \in Y$. Since $y \in \operatorname{Int}\left(\operatorname{St}\left(y, J_{n}\right)^{*}\right)$, there exists $\varepsilon_{y}>0$ such that $N_{\varepsilon_{y}}(y)$ $\subseteq$ St $\left(y, J_{n}\right)^{*}$. The collection $\left\{N_{\varepsilon_{y}}(y): y \in Y\right\}$ covers $Y$. Since $Y$ is compact, there exists a number $\varepsilon^{\prime}>0$ such that every subset of $Y$ of diameter less than $\varepsilon^{\prime}$ will be contained in some member of this cover.

Let $L_{n+1}=\max _{k \in K_{n}}\left\{\left|H_{n}(k)\right|\right\}+1$.

Pick $\varepsilon<\min \left\{1 /(n+1), \varepsilon^{\prime} /\left(2 L_{n+1}+3\right)\right\}$ and let $J_{n+1}^{\prime}$ be any $\varepsilon$-partitioning of $Y$. Let $J_{n+1}=\left\{\mathrm{Cl}\left(j_{n+1}^{\prime}\right): j_{n+1}^{\prime} \in J_{n+1}^{\prime}\right\}$.

For each $j_{n+1} \in J_{n+1}$ we want to construct a polyhedron in $H_{n}^{*}$. This polyhedron will be denoted by $R_{n+1}\left(j_{n+1}\right)$ and will in fact be contained in $\bigcup_{h \cap h^{\prime} \in E} \gamma\left(h \cap h^{\prime}\right)$.

First we must decide which members of $H_{n}$ the set $R_{n+1}\left(j_{n+1}\right)$ is to meet. Let $j_{n} \in J_{n}$ and let $A_{j_{n}}$ be the collection given in Property 10 of the induction. If $j_{n+1}$ $\in J_{n+1}$ and $j_{n} \cap \mathrm{St}^{k}\left(j_{n+1}, J_{n+1}\right)^{*} \neq \varnothing$, but $j_{n} \cap \mathrm{St}^{k-1}\left(j_{n+1}, J_{n+1}\right)^{*}=\varnothing$, then $R_{n+1}\left(j_{n+1}\right)$ is to meet each member of $\mathrm{St}^{L_{n+1}-k}\left(A_{j_{n}}^{*}, H_{n}\left(R_{n}\left(j_{n}\right)\right)\right)$ and $R_{n+1}\left(j_{n+1}\right)$ 
is to miss every member of $H_{n}\left(R_{n}\left(j_{n}\right)\right)$ not in this collection. From now on we will denote this collection by $\mathrm{St}^{L_{n+1}-k}\left(A_{j_{n}}^{*}\right)$.

If $h \in H_{n}$, then let $J(h)=\left\{j_{n+1} \in J_{n+1}: R_{n+1}\left(j_{n+1}\right)\right.$ is to meet $\left.h\right\}$. Let $h \cap h^{\prime} \in E$. We want to define two functions $\theta_{1}^{h, h^{\prime}}=\theta_{1}^{h^{\prime}, h}$ and $\theta_{2}^{h, h^{\prime}}=\theta_{2}^{h^{\prime}, h}$ from $J(h) \cap J\left(h^{\prime}\right)$ onto collections of 3-cells in Int $\left(\Gamma_{n}\left(h \cap h^{\prime}\right)^{*}\right)$ with the following properties:

1. If $C_{i}\left(h, h^{\prime}\right)$ denotes the range of $\theta_{i}^{h, h^{\prime}}$, then $C_{1}\left(h, h^{\prime}\right) \cup C_{2}\left(h, h^{\prime}\right)$ is a finite simple collection of polyhedral 3-cells such that $\left|J(h) \cap J\left(h^{\prime}\right)\right|=\left|C_{i}\left(h, h^{\prime}\right)\right|$ for $i=1,2$.

2. Each member of $C_{i}\left(h, h^{\prime}\right)$ meets each member of $\Gamma_{n}\left(h \cap h^{\prime}\right)$ in a 3-cell.

3. If $j_{n+1}, j_{n+1}^{\prime} \in J(h) \cap J\left(h^{\prime}\right)$ and $\gamma \in \Gamma_{n}\left(h \cap h^{\prime}\right)$, then $j_{n+1} \cap j_{n+1}^{\prime} \neq \varnothing$ iff for $i, k=1,2, \theta_{i}^{h, h^{\prime}}\left(j_{n+1}\right) \cap \theta_{k}^{h, h^{\prime}}\left(j_{n+1}^{\prime}\right) \cap \gamma$ is a 2-cell. If $j_{n+1} \cap j_{n+1}^{\prime}=\varnothing$, then

$$
\theta_{i}^{h, h^{\prime}}\left(j_{n+1}\right) \cap \theta_{k}^{h, h^{\prime}}\left(j_{n+1}\right)=\varnothing \quad \text { for } i, k=1,2 .
$$

4. Denote $\theta_{i}^{h, h}$ by $\theta_{i}^{h}$ and $C_{i}(h, h)$ by $C_{i}(h)$. If $h \neq h^{\prime}$, then $\theta_{i}^{h}\left(j_{n+1}\right)$ meets exactly one member of $C_{k}\left(h, h^{\prime}\right)$, where $k=1$ or 2 . In particular, $\theta_{i}^{h}\left(j_{n+1}\right) \cap \theta_{k}^{h, h^{\prime}}\left(j_{n+1}\right)$ is a 2-cell.

To construct these collections first let $C_{1}^{\prime}\left(h, h^{\prime}\right) \cup C_{2}^{\prime}\left(h, h^{\prime}\right)$ denote a disjoint collection of polyhedral 3-cells in Int $\left(\Gamma_{n}\left(h, h^{\prime}\right)^{*}\right)$ such that $\left|C_{1}\left(h, h^{\prime}\right)\right|=\left|C_{2}^{\prime}\left(h, h^{\prime}\right)\right|$ $=\left|J(h) \cap J\left(h^{\prime}\right)\right|$ and such that each member of $C_{i}^{\prime}\left(h, h^{\prime}\right)$ meets each member of $\Gamma_{n}\left(h \cap h^{\prime}\right)$ in a 3-cell. Let $C^{\prime}=\bigcup_{h \cap h^{\prime} \neq \varnothing} C_{1}^{\prime}\left(h, h^{\prime}\right) \cup C_{2}^{\prime}\left(h, h^{\prime}\right)$. We will also assume that the members of $C^{\prime}$ are pairwise disjoint. Let $\theta_{i}^{h, h^{\prime}}$ be any one-to-one and onto correspondence between $J(h) \cap J\left(h^{\prime}\right)$ and $C_{i}^{\prime}\left(h, h^{\prime}\right)$. If $j_{n+1} \in J(h) \cap J\left(h^{\prime}\right)$ and $\gamma \in \Gamma_{n}\left(h, h^{\prime}\right)$, then construct a polygonal arc $\alpha$ in Int $(\gamma)$ such that $\alpha$ meets each of $\theta_{1}^{h, h^{\prime}}\left(j_{n+1}\right) \cap \gamma$ and $\theta_{2}^{h, h^{\prime}}\left(j_{n+1}\right) \cap \gamma$ in an endpoint. The arc will meet no members of $C^{\prime}$ other than $\theta_{1}^{h, h^{\prime}}\left(j_{n+1}\right)$ and $\theta_{2}^{h, h^{\prime}}\left(j_{n+1}\right)$. If $j_{n+1}$ and $j_{n+1}^{\prime}$ are distinct members of $J(h) \cap J\left(h^{\prime}\right)$ with a point in common and $\gamma \in \Gamma_{n}\left(h, h^{\prime}\right)$, then construct an arc $\alpha_{i, k}$ in Int $(\gamma)$ such that $\alpha_{i, k}$ meets each of $\theta_{i}^{h, h^{\prime}}\left(j_{n+1}\right) \cap \gamma$ and $\theta_{k}^{h, h^{\prime}}\left(j_{n+1}^{\prime}\right) \cap \gamma$ in an endpoint of $\alpha_{i, k}$. The arc $\alpha_{i, k}$ will meet no members of $C^{\prime}$ other than $\theta_{i}^{h, h^{\prime}}\left(j_{n+1}\right)$ and $\theta_{k}^{h, h^{\prime}}\left(j_{n+1}^{\prime}\right)$. Adjust the collection of arcs so that no two meet. "Fatten" each in such a way that the collection of arcs remains disjoint, and meets $\left(C^{\prime}\right)^{*}$ in exactly two 2-cells. Associate each fattened arc to one of the two members of $C_{i}^{\prime}\left(h, h^{\prime}\right)$ that it meets. A member of $C_{i}\left(h, h^{\prime}\right)$ will be a 3-cell which is the union of a member of $C_{i}^{\prime}\left(h, h^{\prime}\right)$ and the fattened arcs associated with it. Let

$$
C=\bigcup_{h \cap h^{\prime} \neq \varnothing} C_{1}\left(h, h^{\prime}\right) \cup C_{2}\left(h, h^{\prime}\right) .
$$

Let $R_{n+1}\left(j_{n+1}\right)=\bigcup_{h \cap h^{\prime} \in E} \theta_{1}^{h, h^{\prime}}\left(j_{n+1}\right) \cup \theta_{2}^{h, h^{\prime}}\left(j_{n+1}\right)$. Let

$$
H_{n+1}=\left\{c \cap \gamma: c \in C, \gamma \in \Gamma_{n} \text {, and } c \cap \gamma \neq \varnothing\right\} \text {. }
$$

Note that $\mu\left(H_{n+1}\right)<4 / 2^{n+1}$. Let $K_{n+1}=\left\{R_{n+1}\left(j_{n+1}\right): j_{n+1} \in J_{n+1}\right\}$.

Due to space limitations we will check only a few of the properties at the next step of the induction. 


\section{DIAGRAM}

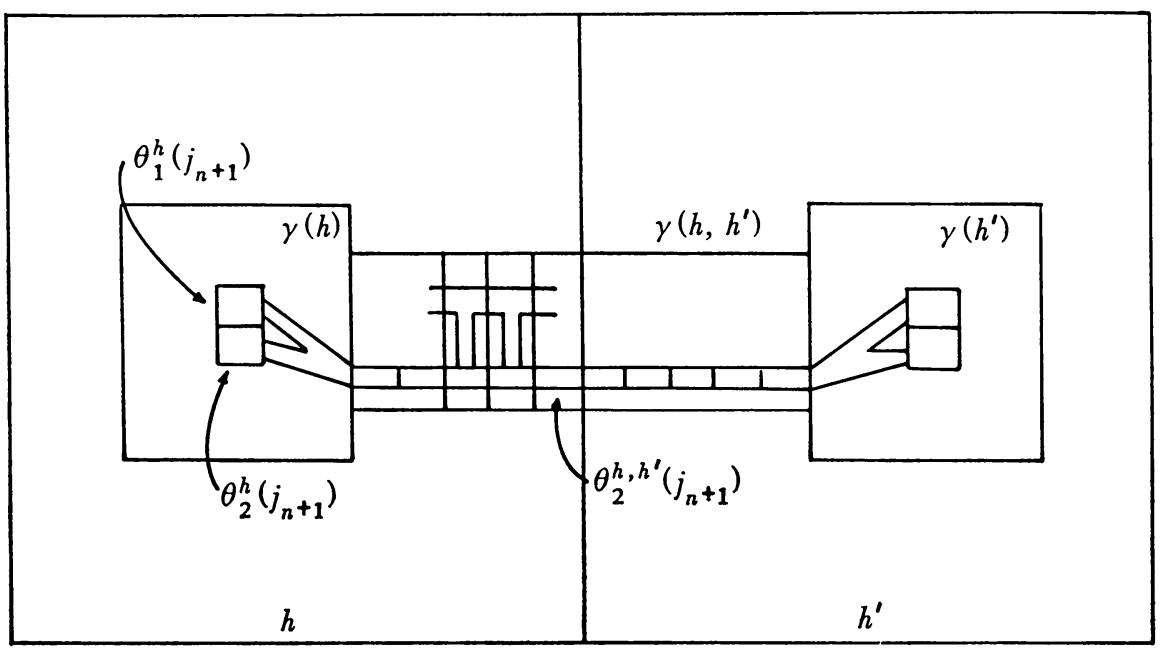

Property 8. Let $j_{n+1} \in J_{n+1}$ and $j_{n} \in J_{n}$ be such that $j_{n} \cap \operatorname{St}\left(j_{n+1}, J_{n+1}\right)^{*} \neq \varnothing$. By the starring rules we know that $R_{n+1}\left(j_{n+1}\right)$ meets every member of $\operatorname{St}^{L_{n+1}-1}\left(A_{j_{n}}^{*}\right)$. Since $R_{n}\left(j_{n}\right)$ is connected and since $L_{n+1} \geqq\left|H_{n}\left(R_{n}\left(j_{n}\right)\right)\right|+1$,

$$
H_{n}\left(R_{n}\left(j_{n}\right)\right)=\mathrm{St}^{L_{n+1}}\left(A_{j_{n}}^{*}\right) .
$$

Therefore, $R_{n+1}\left(j_{n+1}\right)$ meets every member of $H_{n}\left(R_{n}\left(j_{n}\right)\right)$. This stronger version of Property 8 will be needed in the proof of Property 10 .

Since $\mu\left(H_{n}\right)<4 / 2^{n}$ and since $R_{n+1}\left(j_{n+1}\right)$ meets every member of $H_{n}\left(R_{n}\left(j_{n}\right)\right)$, we see that $R_{n}\left(j_{n}\right) \subseteq N_{4 / 2^{n}}\left(R_{n+1}\left(j_{n+1}\right)\right)$.

Property 5. Let $j_{n+1} \in J_{n+1}$ and let $j_{n}^{1}, \ldots, j_{n}^{r}$ be all the members of $J_{n}$ which meet $\mathrm{St}^{L_{n+1}+1}\left(j_{n+1}, J_{n+1}\right)^{*}$. Since $\mu\left(J_{n+1}\right)<\varepsilon$,

$$
d\left[\mathrm{St}^{L_{n+1}+1}\left(j_{n+1}, J_{n+1}\right)^{*}\right]<\left(2\left(L_{n+1}+1\right)+1\right) \varepsilon<\varepsilon^{\prime} .
$$

Thus, there exists $y \in Y$ such that $\mathrm{St}^{L_{n+1}+1}\left(j_{n+1}, J_{n+1}\right)^{*} \subseteq N_{\varepsilon_{y}}(y)$. By the choice of $\varepsilon_{y}$ we know that $y \in j_{n}^{1} \cap \cdots \cap j_{n}^{r}$. Therefore, $j_{n}^{1} \cap \cdots \cap j_{n}^{r} \neq \varnothing$.

Property 6. By the definition of $K_{n+1}, R_{n+1}$ maps $J_{n+1}$ onto $K_{n+1}$. By construction distinct members of $J_{n+1}$ are mapped to distinct members of $K_{n+1}$. Thus, $R_{n+1}$ is one-to-one.

If $j_{n+1}, j_{n+1}^{\prime} \in J_{n+1}$ and $j_{n+1} \cap j_{n+1}^{\prime}=\varnothing$, then $R_{n+1}\left(j_{n+1}\right) \cap R_{n+1}\left(j_{n+1}^{\prime}\right)=\varnothing$. If $j_{n+1} \cap j_{n+1}^{\prime} \neq \varnothing$, then pick $j_{n} \in J_{n}$ such that $j_{n+1} \cap j_{n+1}^{\prime} \cap j_{n} \neq \varnothing$. In Property 8 we showed that each of $R_{n+1}\left(j_{n+1}\right)$ and $R_{n+1}\left(j_{n+1}^{\prime}\right)$ will meet every member of $H_{n}\left(R_{n}\left(j_{n}\right)\right)$. Let $h$ be any member of $H_{n}\left(R_{n}\left(j_{n}\right)\right)$. Since $j_{n+1} \cap j_{n+1}^{\prime} \neq \varnothing, \theta_{1}^{h}\left(j_{n+1}\right)$ $\cap \theta_{1}^{h}\left(j_{n+1}^{\prime}\right) \neq \varnothing$. Therefore, $R_{n+1}\left(j_{n+1}\right) \cap R_{n+1}\left(j_{n+1}^{\prime}\right) \neq \varnothing$.

If $j_{n+1} \cap j_{n+1}^{\prime} \neq \varnothing$, then we will show that $R_{n+1}\left(j_{n+1}\right) \subseteq N_{16 / 2^{n+1}}\left(R_{n+1}\left(j_{n+1}^{\prime}\right)\right)$. Let $x \in R_{n+1}\left(j_{n+1}\right)$. There exist $h \in H_{n}$ which contains $x$, and $j_{n} \in J_{n}$ such that $h \subseteq R_{n}\left(j_{n}\right)$. If $h \in A_{j_{n}}$, then let $j_{n}^{\prime}$ be a member of $J_{n}$ such that $j_{n+1} \cap j_{n+1}^{\prime} \cap j_{n}^{\prime} \neq \varnothing$. 
By Property 8, we know that $R_{n+1}\left(j_{n+1}^{\prime}\right)$ meets every member of $H_{n}\left(R_{n}\left(j_{n}^{\prime}\right)\right)$. Since $R_{n+1}\left(j_{n+1}\right) \cap R_{n}\left(j_{n}\right) \neq \varnothing$, we know by 7 that $j_{n} \cap \mathrm{St}^{L_{n+1}}\left(j_{n+1}, J_{n+1}\right)^{*} \neq \varnothing$. Thus, $j_{n} \cap j_{n}^{\prime} \neq \varnothing$, and $R_{n}\left(j_{n}^{\prime}\right) \cap h \neq \varnothing$. Let $h^{\prime}$ be a member of $H_{n}\left(R_{n}\left(j_{n}^{\prime}\right)\right)$ which meets $h$. Since $R_{n+1}\left(j_{n+1}^{\prime}\right)$ meets $h^{\prime}, d\left[x, R_{n+1}\left(j_{n+1}^{\prime}\right)\right]<d\left[h \cup h^{\prime}\right] \leqq d[h]+d\left[h^{\prime}\right]<16 / 2^{n+1}$.

If $h \notin A_{j_{n}}$, then there exists an integer $k$ such that $0<k<L_{n+1}$ and $h \in \mathrm{St}^{L_{n+1}-k}\left(A_{j_{n}}^{*}\right)$ and $j_{n} \cap \mathrm{St}^{k}\left(j_{n+1}, J_{n+1}\right)^{*} \neq \varnothing$. Since $j_{n+1} \cup j_{n+1}^{\prime} \neq \varnothing$;

$$
j_{n} \cap \mathrm{St}^{k+1}\left(j_{n+1}^{\prime}, J_{n+1}\right)^{*} \neq \varnothing
$$

Therefore, $R_{n+1}\left(j_{n+1}^{\prime}\right)$ meets every member of $\mathrm{St}^{L_{n+1}-k-1}\left(A_{j_{n}}^{*}\right)$. This collection will be nonempty because $L_{n+1}-k-1 \geqq 0$. The remainder of the proof is the same as when $h \in A_{j_{n}}$. Therefore, $R_{n+1}\left(j_{n+1}\right) \subseteq N_{16 / 2^{n+1}}\left(R_{n+1}\left(j_{n+1}^{\prime}\right)\right)$.

Property 4. The members of $K_{n+1}$ have disjoint nonempty interiors by construction. We must show that the interiors are connected.

LEMMA. If $L$ is a finite collection of closed subsets and $A \subseteq L^{*}$, then $A$ is connected provided:

1. $L^{*}$ is connected.

2. $A \cap h$ is connected for all $h \in L$.

3. If $h, h^{\prime} \in L$ and $h \cap h^{\prime} \neq \varnothing$; then $A \cap h \cap h^{\prime} \neq \varnothing$.

Proof. The proof is routine and thus is omitted.

Let $j_{n+1} \in J_{n+1}$. Let $j_{n}$ be a member of $J_{n}$ which meets $j_{n+1}$. We know that $R_{n}\left(j_{n}\right)$ is connected by our induction assumption. We showed in Property 8 that $R_{n+1}\left(j_{n+1}\right)$ meets every member of $H_{n}$ in $R_{n}\left(j_{n}\right)$. To apply the lemma, let $L=H_{n}\left(R_{n}\left(j_{n}\right)\right)$ and $A=R_{n+1}\left(j_{n+1}\right) \cap R_{n}\left(j_{n}\right)$. Therefore, $R_{n+1}\left(j_{n+1}\right) \cap R_{n}\left(j_{n}\right)$ is connected.

If $j_{n}^{\prime} \in J_{n}$ and $R_{n+1}\left(j_{n+1}\right) \cap R_{n}\left(j_{n}^{\prime}\right) \neq \varnothing$, then there exists an integer $k$ such that $R_{n+1}\left(j_{n+1}\right)$ meets exactly those members of $H_{n}\left(R_{n}\left(j_{n}^{\prime}\right)\right)$ in $\mathrm{St}^{k}\left(A_{j_{n}^{\prime}}^{*}\right)$. If $h \in A_{j_{n}^{\prime}}$, then $\mathrm{St}^{k}(h)^{*}$ is connected because the star of a connected set is connected if the links are connected. To apply the lemma let $L=\operatorname{St}^{k}(h)$ and $A=R_{n+1}\left(j_{n+1}\right) \cap L^{*}$. Therefore, $R_{n+1}\left(j_{n+1}\right) \cap L^{*}$ is connected. Since $R_{n}\left(j_{n}\right) \cap h \neq \varnothing, R_{n+1}\left(j_{n+1}\right)$ $\cap\left(R_{n}\left(j_{n}\right) \cup R_{n}\left(j_{n}^{\prime}\right)\right)$ is connected. Therefore, $R_{n+1}\left(j_{n+1}\right)$ is connected. Since distinct members of $H_{n+1}$ meet in 2-cells, Int $\left(R_{n+1}\left(j_{n+1}\right)\right)$ is also connected. Therefore, $K_{n+1}$ is a simple collection.

Property 9. Since most of the properties of a $B$-defining sequence are obvious, we will only check the interlacing axiom and the fact that $H_{n+1}^{*}$ is connected.

Let $h \in H_{n}$. Pick $j_{n} \in J_{n}$ so that $h \subseteq R_{n}\left(j_{n}\right)$. There exists an integer $k$ such that $h \in \mathrm{St}^{L_{n+1}-k}\left(A_{j_{n}}^{*}\right)-\mathrm{St}^{L_{n+1}-k-1}\left(A_{j_{n}}^{*}\right)$. Therefore, if $j_{n+1} \in J_{n+1}$, then $R_{n+1}\left(j_{n+1}\right)$ $\cap h \neq \varnothing$ if and only if $j_{n} \cap \mathrm{St}^{k}\left(j_{n+1}, J_{n+1}\right)^{*} \neq \varnothing$. Note that $j_{n} \cap \cap \mathrm{St}^{k}\left(j_{n+1}, J_{n+1}\right)^{*}$ $\neq \varnothing$ if and only if $j_{n+1} \in \mathrm{St}^{k+1}\left(j_{n}, J_{n+1}\right)$. Therefore, $J(h)=\operatorname{St}^{k+1}\left(j_{n}, J_{n+1}\right)$. Since $j_{n}$ is connected and the members of $J_{n+1}$ are connected, $J(h)^{*}$ is connected. Since $\theta_{i}^{h}$ maps $J(h)$ onto $C_{i}(h)$ with the property that $j_{n+1} \cap j_{n+1}^{\prime} \neq \varnothing$ if and only if 
$\theta_{i}^{n}\left(j_{n+1}\right) \cap \theta_{i}^{n}\left(j_{n+1}^{\prime}\right) \neq \varnothing, C_{i}(h)^{*}$ is also connected for $i=1,2$. Moreover, since $\theta_{1}^{h}\left(j_{n+1}\right) \cap \theta_{2}^{h}\left(j_{n+1}\right) \neq \varnothing$ for each $j_{n+1} \in J(h), C_{1}(h)^{*} \cup C_{2}(h) *$ is connected.

Let $Z$ denote $Z_{H_{n+1}}(h)$. If $z \in Z$, then there exists a unique $h^{\prime} \in H_{n}$ different from $h$ such that $z \cap h^{\prime} \neq \varnothing$. In fact, $z$ is contained in exactly one member of $C_{1}\left(h, h^{\prime}\right)$ $\cup C_{2}\left(h, h^{\prime}\right)$. Denote this member by $C^{\prime}(z)$.

Let $Z_{1}$ and $Z_{2}$ denote two disjoint subcollections of $Z$ such that $Z_{1} \cup Z_{2}=Z$. Let $W_{1}=C_{1}(h)^{*} \cup\left(\cup_{z \in Z_{1}} C(z) \cap h\right)$ and $W_{2}=C_{2}(h)^{*} \cup\left(\cup_{z \in Z_{2}} C(z) \cap h\right)$. Since each $C(z) \cap h$ is a 3-cell and meets each of $C_{i}(h)^{*}, i=1,2$, in a 2-cell, both $W_{1}$ and $W_{2}$ are connected. Since $C_{1}(h) \cup C_{2}(h)$ is a simple collection and since Int $(C(z)) \cap$ Int $\left(C\left(z^{\prime}\right)\right)=\varnothing$ if $z \neq z^{\prime}$, the collection $\left\{W_{1}, W_{2}\right\}$ is simple. In the terminology of [2] $\left\{W_{\mathrm{I}}, W_{2}\right\}$ is a simple complete amalgam of $H_{n+1}(h)$ such that $Z_{i}^{*} \subseteq W_{i}$. Therefore, $H_{n+1}(h)$ is interlaced in $h$.

Since $H_{n+1}(h)^{*}$ is connected for all $h \in H_{n}$, and since $H_{n}^{*}$ is connected, the lemma used in Property 4 tells us that $H_{n+1}^{*}$ is connected.

Property 10. Let $j_{n+1} \in J_{n+1}$. Pick $j_{n} \in J_{n}$ so that $j_{n+1} \cap j_{n} \neq \varnothing$. If $j_{n+1}^{\prime} \in J_{n+1}$ and $j_{n+1} \cap j_{n+1}^{\prime} \neq \varnothing$; then $j_{n} \cap \mathrm{St}\left(j_{n+1}^{\prime}, J_{n+1}\right)^{*} \neq \varnothing$. Thus, by Property $8, R_{n+1}\left(j_{n+1}^{\prime}\right)$ meets every member of $H_{n}\left(R_{n}\left(j_{n}\right)\right)$. In particular, $R_{n+1}\left(j_{n+1}^{\prime}\right)$ meets every member of $A_{j_{n}}$. If $h \in A_{j_{n}}$ and $j_{n+1}^{\prime}$ meets $j_{n+1}$, then by the construction $\theta_{i}^{h}\left(j_{n+1}\right) \cap \theta_{i}^{h}\left(j_{n+1}^{\prime}\right)$ $\neq \varnothing$. Thus, $\theta_{i}^{h}\left(j_{n+1}\right)$ meets $R_{n+1}\left(j_{n+1}^{\prime}\right)$. The desired collection is

$$
A_{j_{n+1}}=\left\{\theta_{i}^{h}\left(j_{n+1}\right): h \in A_{j_{n}} \text { and } i=1,2\right\} \text {. }
$$

Note that $A_{j_{n+1}}^{*} \subseteq A_{j_{n}}^{*}$. We have now completed the proof of Proposition 4 .

Let $K$ be the class of all 1-dimensional continuous curves up to topological equivalence. Let $M$ be the subclass of $K$ consisting of those elements of $K$ having no local cut points. Anderson [8] proved that a necessary and sufficient condition for a member of $M$ to be the universal curve is that it contain no open subset imbeddable in the plane.

Definition. A map will be called monotone if each point-inverse set is compact and connected.

THEOREM 1. There exists a monotone open map of the universal curve onto any continuous curve such that each point-inverse set is also a universal curve.

REMARK. Since the monotone image of a 2 -sphere is a cactoid [1, p. 172], the monotone image of a compact subset of the plane will always have dimension less than or equal to two.

Proof. Let $Y$ be any continuous curve: We can find sequences of continua $\left\{J_{n}\right\}_{n=1}^{\infty},\left\{K_{n}\right\}_{n=1}^{\infty}$, and $B$-defining sequence $\left\{H_{n}\right\}_{n=1}^{\infty}$ with the ten properties stated in Proposition 4. Let $M=\bigcap_{n=1}^{\infty} H_{n}^{*}=\bigcap_{n=1}^{\infty} K_{n}^{*}$. Proposition 3 and Proposition 4 now combine to give an open map $g$ of $M$ onto $Y$. Since $\left\{H_{n}\right\}_{n=1}^{\infty}$ is a $B$-defining sequence, $M$ is homeomorphic to the universal curve. Since the members of $K_{n}$ are connected, $g$ will be monotone. 
To show that each point-inverse set is a universal curve, it is sufficient to show that for each $y \in Y, g^{-1}(y)$ is locally connected, $g^{-1}(y)$ has no local cut points, and $g^{-1}(y)$ contains no open subset imbeddable in the plane $\left({ }^{2}\right)$.

Lemma 1. If $j_{n+3} \in J_{n+3}, j_{n+2} \in J_{n+2}$, and $j_{n+2} \cap j_{n+3} \neq \varnothing$, then $R_{n+3}\left(j_{n+3}\right)$ $=R_{n+3}\left(j_{n+3}\right) \cap R_{n+2}\left(j_{n+2}\right) \cup T_{1} \cup \ldots \cup T_{\text {s }}$ where

1. each $T_{i}$ is a connected union of members of $H_{n+3}$,

2. $T_{i} \cap R_{n+3}\left(j_{n+3}\right) \cap R_{n+2}\left(j_{n+2}\right) \neq \varnothing$ for $i=1, \ldots, s$,

3. the set $\mathrm{St}^{5}\left(T_{i}, H_{n+2}\right)$ * is contained in five members of $H_{n+1}$.

LeMmA 2. Let $j_{n+i} \in J_{n+i}$ for $i=2, \ldots, m$. If $j_{n+2} \cap \cdots \cap j_{n+m} \neq \varnothing$, then $R_{n+m}\left(j_{n+m}\right)=R_{n+m}\left(j_{n+m}\right) \cap \cdots \cap R_{n+2}\left(j_{n+2}\right) \cup S_{1} \cup \cdots \cup S_{r}$, where

1. each $S_{i}$ is connected,

2. each $S_{i}$ meets $R_{n+m}\left(j_{n+m}\right) \cap \cdots \cap R_{n+2}\left(j_{n+2}\right)$,

3. each $S_{i}$ lies in five members of $H_{n+1}$.

Proof. The proof of Lemma 2 follows from Lemma 1 and the construction.

We now will show that $g^{-1}(y)$ is locally connected for all $y \in Y$. The proof given here follows the technique of [3].

Let $x \in g^{-1}(y)$ and let $V$ be an open subset of $g^{-1}(y)$ containing $x$. It is sufficient to show that $x$ is interior to the component of $V$ containing $x$.

Pick $n$ large enough so that $\operatorname{St}^{3}\left(x, H_{n}\right)^{*} \cap g^{-1}(y) \subseteq V$. Let $\rho=\operatorname{St}\left(x, H_{n}\right)^{*}$. If $h, h^{\prime} \in H_{n}$, then we will show that there is at most one component of $g^{-1}(y)$ $\cap \gamma\left(h \cap h^{\prime}\right)$ which meets every member of $\Gamma_{n}\left(h \cap h^{\prime}\right)$. This will prove that there are only a finite number of components of $g^{-1}(y) \cap\left(\operatorname{St}\left(\rho, H_{n}\right)^{*} \cup \bigcup_{\rho \cap h \neq \varnothing} \gamma\left(h \cap h^{\prime}\right)\right)$ meeting $\rho$. Therefore, $V$ has only a finite number of components meeting $\rho$.

Let $\left\{f_{n}\right\}_{n=1}^{\infty}$ be a nested sequence of sets given by the conclusion of Proposition 3 such that $\bigcap_{n=1}^{\infty} f_{n}=g^{-1}(y)$.

Let $h \cap h^{\prime} \in E$, where $h \neq h^{\prime}$. If $R_{n+1}\left(j_{n+1}\right)$ meets $\gamma\left(h \cap h^{\prime}\right)$, then $R_{n+1}\left(j_{n+1}\right)$ $\cap \gamma\left(h \cap h^{\prime}\right)=\theta_{1}^{h, h^{\prime}}\left(j_{n+1}\right) \cup \theta_{2}^{h, h^{\prime}}\left(j_{n+1}\right) \cap \gamma\left(h \cap h^{\prime}\right)$. From now on we will denote $\theta_{i}^{h, h^{\prime}}\left(j_{n+1}\right)$ by $\theta_{i}\left(j_{n+1}\right)$. Note that $f_{n+1} \cap \gamma\left(h \cap h^{\prime}\right)$ is connected.

Let $\gamma(h)=\gamma_{1}, \gamma_{2}, \ldots, \gamma_{k}=\gamma\left(h^{\prime}\right)$ be the members of $\Gamma_{n}\left(h \cap h^{\prime}\right)$ listed so that $\gamma_{i} \cap \gamma_{j} \neq \varnothing$ if and only if $|i-j| \leqq 1$.

Let $F$ denote any continuum in $H_{n+1}^{*}$ which is not contained in $\gamma\left(h \cap h^{\prime}\right)$. If $S$ is a component of $F \cap \gamma\left(h \cap h^{\prime}\right)$ meeting $\gamma_{i}$, then note that $S$ will either meet the interior of every member of $\left\{\gamma_{1}, \ldots, \gamma_{i}\right\}$ or the interior of every member of $\left\{\gamma_{i}, \ldots, \gamma_{k}\right\}$.

Suppose $f_{n+m} \cap \gamma\left(h \cap h^{\prime}\right)$ contains two components $K_{1}$ and $K_{2}$ which meet every member of $\Gamma_{n}\left(h \cap h^{\prime}\right)$. Let $h_{n+m}$ be a member of $H_{n+m}$ contained in $K_{1} \cap \gamma_{25}$. Pick $j_{n+m} \in J_{n+m}$ such that $h_{n+m} \subseteq R_{n+m}\left(j_{n+m}\right)$. We can find $j_{n+i} \in J_{n+i}$ for $i=2$, $\ldots, m-1$, such that $j_{n+m} \cap j_{n+m-1} \cap \cdots \cap j_{n+2} \neq \varnothing$. Therefore, by Lemma 2,

${ }^{(2)}$ The author is grateful to Professor Anderson for suggesting this approach. 
we can find a connected subset of $T$ of $R_{n+m}\left(j_{n+m}\right) \cap \gamma\left(h \cap h^{\prime}\right)$ which contains $h_{n+m}$ and meets $\left(R_{n+m}\left(j_{n+m}\right) \cap \cdots \cap R_{n+2}\left(j_{n+2}\right)\right) \cap\left(\gamma_{21} \cup \cdots \cup \gamma_{29}\right)$.

Let $h_{n+m}^{1}$ be a member of $H_{n+m}$ contained in $R_{n+m}\left(j_{n+m}\right) \cap \cdots \cap R_{n+2}\left(j_{n+2}\right)$ which meets $T$. Note that $h_{n+m}^{1} \subseteq K_{1} \cap\left(\gamma_{20} \cup \cdots \cup \gamma_{30}\right)$. Since $A_{j_{n+2}}^{*} \cap \gamma\left(h \cap h^{\prime}\right)$ $=\varnothing$, we know that $R_{n+m}\left(j_{n+m}\right) \cap \cdots \cap R_{n+2}\left(j_{n+2}\right)$ is not contained in $\gamma\left(h \cap h^{\prime}\right)$. Since $R_{n+m}\left(j_{n+m}\right) \cap \cdots \cap R_{n+2}\left(j_{n+2}\right)$ is connected, we know that the component of $\left(R_{n+m}\left(j_{n+m}\right) \cap \cdots \cap R_{n+2}\left(j_{n+2}\right)\right) \cap \gamma\left(h \cap h^{\prime}\right)$ containing $h_{n+m}^{1}$, denoted by $K_{1}^{\prime}$, either meets every member of $\left\{\gamma_{1}, \ldots, \gamma_{20}\right\}$ or $\left\{\gamma_{30}, \ldots, \gamma_{k}\right\}$. Since $\left|\Gamma_{n}\left(h \cap h^{\prime}\right)\right|>50$, both collections contain at least twenty members so that we can assume $K_{1}^{\prime}$ meets every member of $\left\{\gamma_{1}, \ldots, \gamma_{20}\right\}$. Since $K_{2}$ meets every member of $\Gamma_{n}\left(h \cap h^{\prime}\right)$, $K_{2}$ meets $\gamma_{11}$.

Let $h_{n+m}^{2}$ be a member of $H_{n+m}$ contained in $K_{2} \cap \gamma_{11}$. Choose $j_{n+m}^{\prime} \in J_{n+m}$ and $j_{n+m-1}^{\prime} \in J_{n+m-1}$ so that $h_{n+m}^{2} \subseteq R_{n+m}\left(j_{n+m}^{\prime}\right) \cap R_{n+m-1}\left(j_{n+m-1}^{\prime}\right)$. Since $R_{n+m}\left(j_{n+m}^{\prime}\right)$ meets every member of $H_{n+m-1}$ in $R_{n+m-1}\left(j_{n+m-1}\right)$ that $R_{n+m}\left(j_{n+m}\right)$ does (or vice versa), we know that $K_{2}$ contains a member of $H_{n+m}\left(R_{n+m}\left(j_{n+m}\right)\right)$ in $\gamma_{10} \cup \gamma_{11}$ $\cup \gamma_{12}$. Thus, applying Lemma 2 as before, we can find $h_{n+m}^{3} \in H_{n+m}$ such that $h_{n+m}^{3} \subseteq K_{2} \cap R_{n+m}\left(j_{n+m}\right) \cap \cdots \cap R_{n+2}\left(j_{n+2}\right) \cap\left(\gamma_{5} \cup \cdots \cup \gamma_{17}\right)$. Therefore, for some $\gamma_{i} \in\left\{\gamma_{5}, \ldots, \gamma_{17}\right\}$ there exists two members $h_{n+1}$ and $h_{n+1}^{\prime}$ of $H_{n+1}$ such that $h_{n+1} \cup h_{n+1}^{\prime} \subseteq \gamma_{i}, K_{1} \cap R_{n+m}\left(j_{n+m}\right) \cap \cdots \cap R_{n+2}\left(j_{n+2}\right) \cap h_{n+1} \neq \varnothing$, and

$$
K_{2} \cap R_{n+m}\left(j_{n+m}\right) \cap \cdots \cap R_{n+2}\left(j_{n+2}\right) \cap h_{n+1}^{\prime} \neq \varnothing .
$$

Since $h_{n+1} \cup h_{n+1}^{\prime} \subseteq \gamma_{i} \cap f_{n+1}$, we know by the rules of construction that $h_{n+1}$ $\cap h_{n+1}^{\prime} \neq \varnothing$. Thus, $R_{n+m}\left(j_{n+m}\right) \cap \cdots \cap R_{n+2}\left(j_{n+2}\right) \cap\left(h_{n+1} \cup h_{n+1}^{\prime}\right)$ is connected and $K_{1} \cap K_{2} \neq \varnothing$. This contradicts the assumption that $K_{1}$ and $K_{2}$ are distinct components. Therefore, $f_{n+m} \cap \gamma\left(h \cap h^{\prime}\right)$ contains at most one component which meets every member of $\Gamma_{n}\left(h \cap h^{\prime}\right)$, and $g^{-1}(y)$ is locally connected.

The set $g^{-1}(y)$ has no local cut points. Let $x \in g^{-1}(y)$ and let $U$ be a connected open subset of $g^{-1}(y)$ containing $x$. Suppose $U-\{x\}$ is not connected. Let $U_{1}$ and $U_{2}$ be two different components of $U-\{x\}$. Let $u_{1} \in U_{1}$ and $u_{2} \in U_{2}$. Choose $n$ large enough that $\operatorname{St}^{2}\left(x, H_{n}\right)^{*} \cap g^{-1}(y) \subseteq U-\left\{u_{1}, u_{2}\right\}$.

Choose $h$ and $h^{\prime}$ in $H_{n}$ and $j_{n+1} \in J_{n+1}$ such that $x \in \theta_{i}^{n, h^{\prime}}\left(j_{n+1}\right)$. There is no loss of generality in assuming that $x \in \operatorname{Int}\left(\theta_{1}^{h, h^{\prime}}\left(j_{n+1}\right)\right)$.

Let $S_{1}=\theta_{1}^{h, h^{\prime}}\left(j_{n+1}\right)$.

LEMMA 3. If $m>n$, then there exists a function $T_{m}$ of $H_{m}$ into $H_{m}$ with the following properties:

1. If $h_{m}, h_{m}^{\prime} \in H_{m}$ and $h_{m} \cap h_{m}^{\prime} \neq \varnothing$, then $T_{m}\left(h_{m}\right) \cap T_{m}\left(h_{m}^{\prime}\right) \neq \varnothing$.

2. If $h_{m} \in H_{m}, h_{m+1} \in H_{m+1}$ and $h_{m+1} \subseteq h_{m}$, then $T_{m+1}\left(h_{m+1}\right) \subseteq T_{m}\left(h_{m}\right)$.

3. If $h_{m} \in H_{m}$, then $h_{m}$ and $T_{m}\left(h_{m}\right)$ lie in the same member of $K_{m}$.

4. If $h_{m} \in \mathrm{St}^{k}\left(A_{j_{m}}^{*}\right)$, then $T_{m}\left(h_{m}\right) \in \mathrm{St}^{k}\left(A_{j_{m}}^{*}\right)$ for all $j_{m} \in J_{m}$.

5. If $h_{m} \subseteq \operatorname{St}\left(S_{1}, H_{n}\right)^{*}$, then $T_{m}\left(h_{m}\right) \subseteq \operatorname{St}\left(S_{1}, H_{n}\right)^{*}$. If $h_{m} \nsubseteq \operatorname{St}\left(S_{1}, H_{n}\right)^{*}$, then $T_{m}\left(h_{m}\right)$ $=h_{m}$.

6. $x \notin T_{m}\left(h_{m}\right)$ for all $h_{m} \in H_{m}$. 
Proof. The proof is left to the reader.

Combining Lemma 3 and Proposition 1 we have a continuous function $f$ from $M$ into $M$ defined by $f\left(\bigcap_{m=n+1}^{\infty} h_{m}\right)=\bigcap_{m=n+1}^{\infty} T_{m}\left(h_{m}\right)$, where the sequence $\left\{h_{m}\right\}_{m=n+1}^{\infty}$ is any nested sequence with $h_{m} \in H_{m}$. Moreover, Properties 3, 5 and 6 in the lemma tell us that

1. $f\left(g^{-1}(y)\right) \subseteq g^{-1}(y)$,

2. $f \mid M-\operatorname{St}\left(S_{1}, H_{n}\right)^{*}=$ identity and $f\left(\operatorname{St}\left(S_{1}, H_{n}\right)^{*} \cap M\right) \subseteq \mathrm{St}\left(S_{1}, H_{n}\right)^{*}$,

3. $x \notin f(M)$.

Let $f_{1}=f \mid g^{-1}(y)$. Note that $f_{1}(U) \subseteq U$. Moreover, since $u_{1}$ and $u_{2}$ are not contained in St $\left(S_{1}, H_{n}\right)^{*}, f_{1}\left(u_{1}\right)=u_{1}$ and $f_{1}\left(u_{2}\right)=u_{2}$. Therefore, $f_{1}(U)$ is a connected subset of $U$ which contains both $u_{1}$ and $u_{2}$. But $x \notin f_{1}(U)$ which contradicts the assumption that $U_{1}$ and $U_{2}$ are distinct components of $U-\{x\}$. Therefore, $g^{-1}(y)$ has no local cut points.

The set $g^{-1}(y)$ is not locally imbeddable in the plane. Let $U$ be any nonempty open subset of $g^{-1}(y)$ and let $x \in U$. Pick $n$ large enough so that $\operatorname{St}^{2}\left(x, H_{n}\right)^{*}$ $\cap g^{-1}(y) \subseteq U$. For each integer $m>n$ choose $j_{m} \in J_{m}$ so that $y \in j_{m}$. Since $j_{m+1} \cap j_{m}$ $\neq \varnothing, R_{m+1}\left(j_{m+1}\right)$ meets every member of $H_{m}$ in $R_{m}\left(j_{m}\right)$. Therefore, the same argument as that used to show that $\left\{H_{n}\right\}_{n=1}^{\infty}$ is a $B$-defining sequence can be used to show that $\left\{H_{m}\left(R_{m}\left(j_{m}\right) \cap \cdots \cap R_{n+1}\left(j_{n+1}\right)\right)\right\}_{m=n+1}^{\infty}$ is a $B$-defining sequence. Thus, $X=\bigcap_{m=n+1}^{\infty} R_{m}\left(j_{m}\right)$ is homeomorphic to the universal curve. Note that $X$ is contained in $g^{-1}(y)$. Since $x \in g^{-1}(y)$, we know by the construction that $R_{n+1}\left(j_{n+1}\right)$ $\cap \mathrm{St}^{2}\left(x, H_{n}\right)^{*} \neq \varnothing$. Therefore, $X \cap \mathrm{St}^{2}\left(x, H_{n}\right)^{*} \neq \varnothing$ and thus $X \cap U \neq \varnothing$. Since the universal curve is locally not imbeddable in the plane, $U$ is not imbeddable in the plane.

Proposition 5. If $Y$ is any nondegenerate continuous curve, then there exist two sequences of finite collections of compacta $J=\left\{J_{n}\right\}_{n=1}^{\infty}$ and $K=\left\{K_{n}\right\}_{n=1}^{\infty}$ with the following properties:

1. $J_{1}=\{Y\}$ and $J_{n}^{*}=Y$ for all $n$.

2. $K_{1}=\left\{I^{3}\right\}$ and $K_{n}^{*} \subseteq K_{n-1}^{*}$ for all $n$.

3. $\mu\left(J_{n}\right)<1 / n$.

4. $J_{n}$ and the collection of components of members of $K_{n}$ are simple collections.

5. There exists an integer $L_{n}>1$ with the property that if $j_{n} \in J_{n}$ and $j_{n-1}^{1}, \ldots, j_{n-1}^{r}$ are all the members of $J_{n-1}$ which meet $\operatorname{St}^{L_{n}+1}\left(j_{n}, J_{n}\right)^{*}$, then $j_{n-1}^{1} \cap \cdots \cap j_{n-1}^{r} \neq \varnothing$.

6. There exists a one-to-one and onto correspondence between $J_{n}$ and $K_{n}$ given by $R_{n}$ such that $R_{n}\left(j_{n}\right) \cap R_{n}\left(j_{n}^{\prime}\right) \neq \varnothing$ if and only if $j_{n} \cap j_{n}^{\prime} \neq \varnothing$. If $j_{n} \cap j_{n}^{\prime} \neq \varnothing$, then $R_{n}\left(j_{n}\right) \subseteq N_{8 / 2^{n-1}}\left(R_{n}\left(j_{n}^{\prime}\right)\right)$.

7. If $j_{n} \in J_{n}$ and $j_{n-1} \in J_{n-1}$, then $R_{n}\left(j_{n}\right) \cap R_{n-1}\left(j_{n-1}\right) \neq \varnothing$ if and only if $j_{n-1}$ meets $\operatorname{St}^{L_{n}}\left(j_{n}, J_{n}\right)^{*}$. Also if $k_{n} \in K_{n}, k_{n-1} \in K_{n-1}$, and $k_{n} \cap k_{n-1} \neq \varnothing$, then $k_{n}$ $\cap \operatorname{Int}\left(k_{n-1}\right) \neq \varnothing$.

8. If $j_{n} \in J_{n}, j_{n-1} \in J_{n-1}$ and $j_{n-1} \cap j_{n} \neq \varnothing$, then $R_{n}\left(j_{n}\right)$ meets every member of $H_{n-1}\left(R_{n-1}\left(j_{n-1}\right)\right)$, and $R_{n-1}\left(j_{n-1}\right) \subseteq N_{8 / 2^{n}}\left(R_{n}\left(j_{n}\right)\right)$. 
9. There exists a finite simple collection of polyhedral 3-cells $H_{n}$ such that $H_{n}$ refines $K_{n}, K_{n}^{*}=H_{n}^{*}$, and $H=\left\{H_{n}\right\}_{n=1}^{\infty}$ is a B-defining sequence. Also, $\mu\left(H_{n}\right)<4 / 2^{n}$. Distinct members of $H_{n}$ meet in the empty set or a 2-cell.

10. For each $j_{n} \in J_{n}$ there exists a collection $A_{j_{n}} \subseteq H_{n}$ such that $A_{j_{n}}^{*} \subseteq R_{n}\left(j_{n}\right)$ and such that if $j_{n}^{\prime} \in J_{n}$ and $j_{n} \cap j_{n}^{\prime} \neq \varnothing$, then $R_{n}\left(j_{n}^{\prime}\right)$ meets each member of $A_{j_{n}}$. Each component of $R_{n}\left(j_{n}\right)$ will contain exactly one member of $A_{j_{n}}$ and each component of $R_{n}\left(j_{n}^{\prime}\right)$ will meet some member of $A_{j_{n}}$. No member of $H_{n}$ will meet two members of the collection $\left\{a \in A_{j_{n}}: j_{n} \in J_{n}\right\}$.

11. If $j_{n}^{1} \cap \cdots \cap j_{n}^{r} \neq \varnothing$, then the diameter of each component of $R_{n}\left(j_{n}^{1}\right) \cup \cdots$ $\cup R_{n}\left(j_{n}^{r}\right)$ is less than $20 / 2^{n-1}$.

Proof. We can choose the metric on $Y$ so that $d[Y]<1$. Define $R_{1}$ between $J_{1}$ and $K_{1}$ by $R_{1}(Y)=I^{3}$. Since $d[Y]<1, \mu\left(J_{1}\right)<1$.

Let $H_{1}=\left\{I^{3}\right\}$. Since $d\left[I^{3}\right]=3^{1 / 2}<2, \mu\left(H_{1}\right)<4 / 2^{1}$. All of the other conditions in the first stage of the induction are trivially satisfied. Assume the theorem for the integer $n$.

Let $y \in Y$. Since $y \in \operatorname{Int}\left(\operatorname{St}\left(y, J_{n}\right)^{*}\right)$, there exists $\varepsilon_{y}>0$ such that $N_{\varepsilon_{y}}(y)$ $\subseteq$ St $\left(y, J_{n}\right)^{*}$. The collection $\left\{N_{\varepsilon_{y}}(y): y \in Y\right\}$ covers $Y$. Since $Y$ is compact, there exists a number $\varepsilon^{\prime}>0$ such that every subset of $Y$ of diameter less than $\varepsilon^{\prime}$ will be contained in some member of this cover.

Let $L_{n+1}^{\prime}=1+\mid\left\{h \cap h^{\prime}: h, h^{\prime} \in H_{n}\right.$ and $\left.h \cap h^{\prime} \neq \varnothing\right\} \mid$. Let $L_{n+1}=3 L_{n+1}^{\prime}$. Let $y_{1}$ and $y_{2}$ be two distinct points in $Y$. Let $\varepsilon$ be any positive real number such that

$$
e<\min \left\{(n+1)^{-1}, \varepsilon^{\prime} /\left(4 L_{n+1}+3\right), \frac{1}{3} d\left(y_{1}, y_{2}\right)\right\} \text {. }
$$

Let $J_{n+1}^{\prime}$ be an $\varepsilon$-partitioning of $Y$ and $J_{n+1}=\left\{\mathrm{Cl}_{Y}\left(j^{\prime}\right): j^{\prime} \in J_{n+1}^{\prime}\right\}$.

Let $j_{n} \in J_{n}$ and let $A_{j_{n}}$ be the collection given in Property 10 of the induction. If $j_{n+1} \in J_{n+1}$ and $j_{n} \cap \mathrm{St}^{k}\left(j_{n+1}, J_{n+1}\right)^{*} \neq \varnothing$, but $j_{n} \cap \mathrm{St}^{k-1}\left(j_{n+1}, J_{n+1}\right)^{*}=\varnothing$, then $R_{n+1}\left(j_{n+1}\right)$ is to meet exactly those members of $H_{n}\left(R_{n}\left(j_{n}\right)\right)$ in $\operatorname{St}^{L_{n+1}-k}\left(A_{j_{n}}^{*}\right)$.

Since we will need stronger versions of Properties 5 and 8 as well as Property 7 to define the construction of $H_{n+1}$, we mention them now. In the same way that we proved Property 5 in Proposition 4 , we can prove that if $j_{n}^{1}, \ldots, j_{n}^{r}$ are all the members of $J_{n}$ which meet $\mathrm{St}^{2 \cdot L_{n+1}}\left(j_{n+1}, J_{n+1}\right)^{*}$, then $j_{n}^{1} \cap \cdots \cap j_{n}^{r} \neq \varnothing$. Similarly, we can prove that if $j_{n} \cap \mathrm{St}^{2 \cdot L_{n+1}^{\prime}}\left(j_{n+1}, J_{n+1}\right)^{*} \neq \varnothing$, then $R_{n+1}\left(j_{n+1}\right)$ meets every member of $H_{n}\left(R_{n}\left(j_{n}\right)\right)$. Property 7 is the same as before.

Let $h$ and $h^{\prime}$ be members of $H_{n}$ such that $h \cap h^{\prime} \neq \varnothing$. For each such pair we want to define a subcollection of $J_{n+1}$. If $h=h^{\prime}$, then let $J(h)=\left\{j_{n+1}: R_{n+1}\left(j_{n+1}\right)\right.$ is to meet $h$. If $h \neq h^{\prime}$, then $J\left(h \cap h^{\prime}\right)$ will be a subcollection of $J(h) \cap J\left(h^{\prime}\right)$ defined by the two cases below.

Case 1. Let $G_{n}$ be defined exactly as $G_{n}$ is defined in the proof of Proposition 3. If $g \in G_{n}$ and $g=j_{n}^{1} \cup \cdots \cup j_{n}^{r}$, then let

$$
\lambda(g)=\left\{h \cap h^{\prime}: h \cap h^{\prime} \neq \varnothing, h \neq h^{\prime}, h \cup h^{\prime} \subseteq R_{n}\left(j_{n}^{1}\right) \cup \cdots \cup R_{n}\left(j_{n}^{r}\right)\right\} .
$$

Note that $|\lambda(g)|<L_{n+1}^{\prime}$. 
We want to define a collection of subsets $\xi$ with the following properties:

1. The set $\xi$ is contained in $J_{n+1}$.

2. The members of $\xi$ are pairwise disjoint.

3. If $j_{n+1}^{\prime} \in \xi$ and $j_{n+1}^{\prime \prime} \in \mathrm{St}^{2}\left(j_{n+1}^{\prime}, J_{n+1}\right)$, then $R_{n+1}\left(j_{n+1}^{\prime \prime}\right)$ meets exactly those members of $H_{n}$ in $H_{n}\left(R_{n}\left(j_{n}^{1}\right) \cup \cdots \cup R_{n}\left(j_{n}^{r}\right)\right)$.

4. $|\xi|=|\lambda(g)|$.

If $\lambda(g)$ is the empty collection, then let $\xi$ also be the empty collection. If $\lambda(g)$ is nonempty, then let $j_{n+1}$ be a member of $J_{n+1}$ such that $j_{n}^{1} \cap \cdots \cap j_{n}^{r} \cap j_{n+1} \neq \varnothing$. Let $\xi^{\prime}=\left\{j_{n+1}^{0}, \ldots, j_{n+1}^{k}\right\}$ be a simple chain in $J_{n+1}$ such that $j_{n+1}^{0}=j_{n+1}$ and $j_{n+1}^{k}$ $\cap \operatorname{Bd}(g) \neq \varnothing$. The collection $\xi=\left\{j_{n+1}^{0}, j_{n+1}^{2}, \ldots, j_{n+1}^{2 \cdot|\lambda(g)|}\right\}$ will have the desired properties. Let $\phi$ be any one-to-one correspondence from $\xi$ onto $\lambda(g)$. If $j_{n+1} \in \xi$, then let $j_{n+1}$ be a member of $J\left(\phi\left(j_{n+1}\right)\right)$. Note that if $\phi\left(j_{n+1}\right)=h \cap h^{\prime}$, then $j_{n+1}$ $\in J(h) \cap J\left(h^{\prime}\right)$.

Case 2a. Let $h \in A_{j_{n}}$, where $j_{n} \in J_{n}$. If $j_{n+1} \in J(h)$, but there exists $j_{n+1}^{\prime}$ $\in \operatorname{St}\left(j_{n+1}, J_{n+1}\right)-J(h)$, then choose $j_{n}^{\prime} \in J_{n}$ so that $j_{n}^{\prime} \cap j_{n+1} \neq \varnothing$. Since $j_{n}^{\prime} \cap j_{n} \neq \varnothing$, $R_{n}\left(j_{n}^{\prime}\right) \cap h \neq \varnothing$. Let $h^{\prime}$ be a member of $H_{n}\left(R_{n}\left(j_{n}^{\prime}\right)\right)$ which meets $h$. Let $j_{n+1}$ be a member of $J\left(h \cap h^{\prime}\right)$. Since $R_{n+1}\left(j_{n+1}\right)$ meets $h^{\prime}, j_{n+1}$ is also a member of $J(h) \cap J\left(h^{\prime}\right)$.

Case 2b. Let $h \in H_{n}\left(R_{n}\left(j_{n}\right)\right)$, but $h \notin A_{j_{n}}$. Let $h_{1}$ be the member of $A_{j_{n}}$ in the same component of $R_{n}\left(j_{n}\right)$ as $h$. Let $\eta=\left\{h_{1}, \ldots, h_{i-1}, h_{i}\right\}$ be a simple chain of minimal length in $H_{n}\left(R_{n}\left(j_{n}\right)\right)$ such that $h_{i}=h$. Note that $\eta$ has at least two members. If $j_{n+1} \in J(h)$, but there exists $j_{n+1}^{\prime} \in \mathrm{St}\left(j_{n+1}, J_{n+1}\right)-J(h)$, then let $j_{n+1}$ be a member of $J\left(h_{i-1} \cap h\right)$. The chain $\eta$ will be considered fixed for all members of $J(h)$. Since $\eta$ is of minimal length, we know that $j_{n+1} \in J\left(h_{i-1}\right)$.

Let $\Gamma_{n}$ be a simple 1-dimensional collection of 3-cells with the same properties that $\Gamma_{n}$ had in Proposition 4. Also, let $\gamma\left(h \cap h^{\prime}\right)$ be defined as before.

By the same methods as those used in Proposition 4, we can define a simple 1-dimensional collection $C$ of polyhedral 3-cells in $\Gamma_{n}^{*}$ with the following properties:

1. If $h$ and $h^{\prime}$ are members of $H_{n}$ with a point in common, then there exist two one-to-one functions of $J\left(h \cap h^{\prime}\right)$ into $C$, denoted by $\theta_{1}^{h, h^{\prime}}=\theta_{1}^{h^{\prime}, h}$ and $\theta_{2}^{h, h^{\prime}}=\theta_{2}^{h^{\prime}, h}$. If $C_{i}\left(h, h^{\prime}\right)$ denotes the range of $\theta_{i}^{h, h^{\prime}}$, then the members of $C_{i}\left(h, h^{\prime}\right)$ are pairwise disjoint.

2. The set $C_{i}\left(h, h^{\prime}\right)^{*}$ is contained in Int $\left(\Gamma_{n}\left(h \cap h^{\prime}\right)^{*}\right)$.

3. Each member of $C_{i}\left(h, h^{\prime}\right)$ meets each member of $\Gamma_{n}\left(h \cap h^{\prime}\right)$ in a 3-cell.

4. If $j_{n+1} \in J(h)$, then $\theta_{1}^{h}\left(j_{n+1}\right) \cap \theta_{2}^{h}\left(j_{n+1}\right) \neq \varnothing$.

5. Let $C^{\prime}=\bigcup_{h \cap h^{\prime} \neq \varnothing} C_{1}\left(h, h^{\prime}\right) \cup C_{2}\left(h, h^{\prime}\right)$. If $h \neq h^{\prime}$, then $\theta_{i}^{h, h^{\prime}}\left(j_{n+1}\right)$ meets exactly four members of $C^{\prime}$. In particular, $\theta_{i}^{h, h^{\prime}}\left(j_{n+1}\right)$ meets each $\theta_{k}^{h}\left(j_{n+1}\right)$ and $\theta_{k}^{h^{\prime}}\left(j_{n+1}\right)$, where $k=1$, 2. Since $J\left(h \cap h^{\prime}\right) \subseteq J(h) \cap J\left(h^{\prime}\right), \theta_{k}^{h}\left(j_{n+1}\right)$ and $\theta_{k}^{h^{\prime}}\left(j_{n+1}\right)$ will exist.

6. If $j_{n+1}$ and $j_{n+1}^{\prime}$ are distinct members of $J(h)$ such that $j_{n+1} \cap j_{n+1}^{\prime} \neq \varnothing$, then there exist four simple collections of polyhedral 3-cells, $\alpha_{i}^{h}\left(j_{n+1}\right)$ and $\alpha_{i}^{h}\left(j_{n+1}^{\prime}\right)$ where $i=1,2$, with the following properties: 
(a) The set $\alpha_{i}^{h}\left(j_{n+1}\right)^{*}$ is contained in $\gamma(h)$ and $\left|\alpha_{i}^{h}\left(j_{n+1}\right)\right|=2$.

(b) One member of $\alpha_{i}^{h}\left(j_{n+1}\right)$ meets $\theta_{i}^{h}\left(j_{n+1}\right)$ in a 2-cell, and the other meets $\theta_{i}^{h}\left(j_{n+1}^{\prime}\right)$ in a 2-cell. Moreover, $\alpha_{i}^{h}\left(j_{n+1}\right)^{*}$ meets no other members of $C^{\prime}$.

(c) Distinct chains of the form $\alpha_{i}^{h}$ have disjoint underlying point sets.

Let $\alpha\left(j_{n+1}\right)=$ the collection of all 3-cells which are members of some $\alpha_{i}^{h}\left(j_{n+1}\right)$. Let $C=C^{\prime} \cup\left(\bigcup_{j_{n+1} \in J_{n+1}} \alpha\left(j_{n+1}\right)\right)$.

Let $H_{n+1}$ and $K_{n+1}$ be defined as in Proposition 4. Let

$$
R_{n+1}\left(j_{n+1}\right)=\bigcup_{j_{n+1} \in J\left(h \cap h^{\prime}\right)}\left(\theta_{1}^{n, h^{\prime}}\left(j_{n+1}\right) \cup \theta_{2}^{h, h^{\prime}}\left(j_{n+1}\right)\right) \cup \alpha\left(j_{n+1}\right)^{*}
$$

We now have to check that the eleven properties in the induction statement hold for the integer $n+1$.

The first nine properties can be checked in the same way that the first nine properties in Proposition 4 were checked.

Property 10. Let $K$ be a component of $R_{n+1}\left(j_{n+1}\right)$. Let $h$ be any member of $H_{n}$ such that $K \cap \operatorname{Int}(h) \neq \varnothing$. Note that $\theta_{1}^{h}\left(j_{n+1}\right) \subseteq K$. If St $\left(j_{n+1}, J_{n+1}\right) \subseteq J(h)$, then $\theta_{1}^{n}\left(j_{n+1}^{\prime}\right)$ exists for all $j_{n+1}^{\prime} \in \operatorname{St}_{J_{n+1}}\left(j_{n+1}\right)$. But if this is the case, then $R_{n+1}\left(j_{n+1}^{\prime}\right)$ $\cap \theta_{1}^{h}\left(j_{n+1}\right) \neq \varnothing$.

If St $\left(j_{n+1}, J_{n+1}\right) \nsubseteq J(h)$ and $h \in A_{j_{n}}$ for some $j_{n} \in J_{n}$, then by Case 2a, we know that there exists $j_{n}^{\prime} \in J_{n}$ and $h^{\prime} \in H_{n}\left(R_{n}\left(j_{n}^{\prime}\right)\right)$ such that $h^{\prime} \cap h \neq \varnothing, j_{n}^{\prime} \cap j_{n+1} \neq \varnothing$, and $j_{n+1} \in J\left(h \cap h^{\prime}\right)$. Since $j_{n+1} \in J\left(h \cap h^{\prime}\right), \theta_{1}^{h, h^{\prime}}\left(j_{n+1}\right)$ exists. Moreover, $\theta_{1}^{h}\left(j_{n+1}\right)$ $\cup \theta_{1}^{h, h^{\prime}}\left(j_{n+1}\right) \cup \theta_{1}^{h^{\prime}}\left(j_{n+1}\right)$ is a connected subset of $R_{n+1}\left(j_{n+1}\right)$ so that $\theta_{1}^{h^{\prime}}\left(j_{n+1}\right) \subseteq K$. Since St $\left(j_{n+1}, J_{n+1}\right) \subseteq J\left(h^{\prime}\right), \theta_{1}^{h^{\prime}}\left(j_{n+1}\right)$ will be the desired member of $H_{n+1}(K)$.

If St $\left(j_{n+1}, J_{n+1}\right) \nsubseteq J(h)$ and $h \subseteq R_{n}\left(j_{n}\right)$, but $h \notin A_{j_{n}}$, then by Case $2 \mathrm{~b}$, we found a simple chain $\eta=\left\{h_{1}, \ldots, h_{i-1}, h_{i}=h\right\}$ in $H_{n}\left(R_{n}\left(j_{n}\right)\right)$ of minimal length where $h_{1} \in A_{j_{n}}$. By the starring rules we know that $\mathrm{St}\left(j_{n+1}, J_{n+1}\right) \subseteq J\left(h_{i-1}\right)$. As above, $\theta_{1}^{h_{1}-1}\left(j_{n+1}\right)$ will be the desired member of $H_{n+1}(K)$. Pick one such member from each component of $R_{n+1}\left(i_{n+1}\right)$ and denote this collection by $A_{j_{n+1}}$. Note that we can assume that $\bigcup_{j_{n+1} \in J_{n+1}} A_{j_{n+1}}$ is a subcollection of $\theta_{1}=\left\{\theta_{1}^{h}\left(j_{n+1}\right): h \in H_{n}, j_{n+1} \in J(h)\right\}$. Since no member of $H_{n+1}$ meets two members of $\theta_{1}$, no member of $H_{n+1}$ will meet two members of $\left\{a \in A_{j_{n+1}}: j_{n+1} \in J_{n+1}\right\}$.

Property 11. Let $j_{n+1}^{1}, \ldots, j_{n+1}^{s}$ be distinct members of $J_{n+1}$ with a point in common. We want to show that if $K$ is a component of $R_{n+1}\left(j_{n+1}^{1}\right) \cup \ldots$ $\cup R_{n+1}\left(j_{n+1}^{s}\right)$, then $d[K]<5 \cdot 4 / 2^{n}$.

Let $D_{1}=\left\{j_{n+1} \in J_{n+1}: j_{n+1} \in J\left(h \cap h^{\prime}\right)\right.$ under the rules of Case 1 $\}$. Let $D_{2}=\left\{j_{n+1} \in J_{n+1}: j_{n+1} \in J\left(h \cap h^{\prime}\right)\right.$ under rules of Cases $2 \mathrm{a}$ and $\left.2 \mathrm{~b}\right\}$. We want to show that $D_{1}^{*} \cap D_{2}^{*}=\varnothing$. If $j_{n+1}^{\prime \prime} \in \mathrm{St}^{2}\left(D_{1}^{*}, J_{n+1}\right)$, then there exists $g \in G_{n}$ such that $g=j_{n}^{1} \cup \cdots \cup j_{n}^{r}$ and $R_{n+1}\left(j_{n+1}^{\prime \prime}\right)$ meets exactly those members of $H_{n}$ contained in $R_{n}\left(j_{n}^{1}\right) \cup \cdots \cup R_{n}\left(j_{n}^{r}\right)$. Therefore, no member of St $\left(D_{1}^{*}, J_{n+1}\right)$ can be a member of $D_{2}$ and $D_{1}^{*} \cap D_{2}^{*}=\varnothing$. Therefore, the collection $\left\{j_{n+1}^{1}, \ldots, j_{n+1}^{s}\right\}$ does not contain members from both $D_{1}$ and $D_{2}$. Hence, we can consider the two cases separately. 
Case 1. Since the members of $D_{1}$ are pairwise disjoint, only one member of $\left\{j_{n+1}^{1}, \ldots, j_{n+1}^{s}\right\}$ can possibly belong to $D_{1}$. If $j_{n+1}^{i} \in D_{1}$, then exactly one component of $R_{n+1}\left(j_{n+1}^{1}\right) \cup \cdots \cup R_{n+1}\left(j_{n+1}^{s}\right)$ will meet more than one member of $H_{n}$. Namely, the component containing $\theta_{1}^{h}\left(j_{n+1}^{i}\right) \cup \theta_{1}^{h, h^{\prime}}\left(j_{n+1}^{i}\right) \cup \theta_{1_{1}^{\prime}}^{h^{\prime}}\left(j_{n+1}^{i}\right)$, where $h \cap h^{\prime}=\phi\left(j_{n+1}^{i}\right)$. In this case $d[K] \leqq d\left[h \cup h^{\prime}\right]<2 \cdot 4 / 2^{n}$.

Case 2. Suppose $K$ is a component of $R_{n+1}\left(j_{n+1}^{1}\right) \cup \cdots \cup R_{n+1}\left(j_{n+1}^{s}\right)$ and some member of $\left\{j_{n+1}^{1}, \ldots, j_{n+1}^{s}\right\}$ is in $D_{2}$. If $K \cap R_{n}\left(j_{n}\right) \neq \varnothing$, then let $h_{0}$ be a member of $H_{n}\left(R_{n}\left(j_{n}\right)\right)$ such that $h_{0} \in \mathrm{St}^{k}\left(A_{j_{n}}^{*}\right)$ and $K \cap \mathrm{St}^{k-1}\left(A_{j_{n}}^{*}\right)^{*}=\varnothing$.

Note that there does not exist any simple chain of four members $\left\{h_{0}, h_{1}, h_{2}, h_{3}\right\}$ in $H_{n}\left(R_{n}\left(j_{n}\right)\right)$ such that $K \cap h_{i} \neq \varnothing$ for $i=0,1,2,3$ and $K \cap\left(h_{i} \cup h_{i+1}\right)$ is connected. Therefore, if $C$ is a component of $R_{n}\left(j_{n}\right)$, then $K \cap C \subseteq \operatorname{St}^{2}\left(h_{0}, H_{n}\right)^{*}$. If $R_{n+1}\left(j_{n+1}^{i}\right) \cap K \nsubseteq C$, then by Case $2 \mathrm{a}$ we know that there exists $j_{n+1}^{i}$ such that $j_{n}^{i} \cap j_{n+1}^{i} \neq \varnothing$ and $j_{n+1}^{i} \in J\left(h_{0} \cap h^{\prime}\right)$, where $h^{\prime} \in H_{n}\left(R_{n}\left(j_{n}^{i}\right)\right)$. Since $R_{n+1}\left(j_{n+1}^{i}\right)$ meets every member of $H_{n}\left(R_{n}\left(j_{n}^{i}\right)\right), j_{n+1}^{i}$ is not a member of any $J\left(h^{\alpha} \cap h^{\beta}\right)$, where $h^{\alpha}$ $\cup h^{\beta} \subseteq R_{n}\left(j_{n}^{i}\right)$ and $h^{\alpha} \neq h^{\beta}$. Therefore, $K \cap R_{n}\left(j_{n}^{i}\right) \subseteq \mathrm{St}\left(h_{0}, H_{n}\right)^{*}$. Since no member of $H_{n}$ meets two members of $A=\bigcup_{j_{n} \in J_{n}} A_{j_{n}}$, each member of St $\left(h_{0}, H_{n}\right)$ meets no member of $A$ other than $h_{0}$. Therefore, $K \subseteq \mathrm{St}^{2}\left(h_{0}, H_{n}\right)^{*}$ and $d[K]<20 / 2^{n}$. We have now established our theorem.

Definition. A map is called light if each point-inverse set is totally disconnected.

THEOREM 2. There exists a light open map of the universal curve onto any nondegenerate continuous curve such that each point-inverse set is a Cantor set.

REMARK. The above theorem is not true if we replace the universal curve by the plane universal curve. L. F. McAuley [17] showed that there is no light open map from the plane universal curve onto a 2-cell.

Proof. Let $Y$ be any nondegenerate continuous curve. By Proposition 5, we can find sequences of compacta $\left\{J_{n}\right\}_{n=1}^{\infty},\left\{K_{n}\right\}_{n=1}^{\infty}$, and $\left\{H_{n}\right\}_{n=1}^{\infty}$ with the eleven properties stated there. Let $M=\bigcap_{n=1}^{\infty} H_{n}^{*}=\bigcap_{n=1}^{\infty} K_{n}^{*}$. Proposition 3 and Proposition 5 now combine to give an open map $g$ of $M$ onto $Y$. Since $\left\{H_{n}\right\}_{n=1}^{\infty}$ is a $B$-defining sequence, $M$ is homeomorphic to the universal curve. Since the diameters of the components of $R_{n}\left(j_{n}^{1}\right) \cup \cdots \cup R_{n}\left(j_{n}^{r}\right)$, where $j_{n}^{1} \cap \cdots \cap j_{n}^{r} \neq \varnothing$, are less than $20 / 2^{n}, g$ will be light.

Each point-inverse set is a Cantor set. Let $y \in Y$ and $x \in g^{-1}(y)$. Since $g$ is light, it is sufficient to show that every neighborhood of $x$ contains two points of $g^{-1}(y)$. Let $V$ be any neighborhood of $x$ in $g_{:}^{-1}(y)$. Let $\left\{f_{n}\right\}_{n=1}^{\infty}$ be any nested sequence given by the conclusion of Proposition 3 such that $g\left(\bigcap_{n=1}^{\infty} f_{n}\right)=y$. For each $n$, pick $j_{n} \in J_{n}$ such that $y \in j_{n}$ and $R_{n}\left(j_{n}\right) \subseteq f_{n}$. Choose $n$ large enough that $\operatorname{St}^{2}\left(x, H_{n}\right)^{*}$ $\cap g^{-1}(y) \subseteq V$.

Let $h$ be a member of $\operatorname{St}^{2}\left(x, H_{n}\right)$ such that $R_{n+1}\left(j_{n+1}\right)$ meets $h$. Since $R_{n+2}\left(j_{n+2}\right)$ meets every member of $R_{n+1}\left(j_{n+1}\right), R_{n+2}\left(j_{n+2}\right)$ meets both $\theta_{1}^{n}\left(j_{n+1}\right)=h_{1}$ and $\theta_{2}^{h}\left(j_{n+1}\right)=h_{2}$. Hence, $\theta_{1}^{h_{1}}\left(j_{n+2}\right)$ and $\theta_{1}^{h}\left(j_{n+2}\right)$ exist and are disjoint. Since $R_{n+i}\left(j_{n+i}\right)$ meets every member of $R_{n+2}\left(j_{n+2}\right), \bigcap_{i=2}^{\infty} R_{n+i}\left(j_{n+i}\right) \cap \theta_{1}^{h}\left(j_{n+2}\right) \neq \varnothing$. Similarly, 
$\bigcap_{i=2}^{\infty} R_{n+i}\left(j_{n+i}\right) \cap \theta_{1}^{h}\left(j_{n+2}\right) \neq \varnothing$. Therefore, $V$ contains at least two points, and we have established Theorem 2 .

\section{BIBLIOGRAPHY}

1. P. Alexandroff, C. R. Acad. URRS 4 (1936), 293.

2. R. D. Anderson, A characterization of the universal curve and a proof of its homogeneity, Ann. of Math. (2) 67 (1958), 313-324. MR 20 \#2675.

3. — Continuous collections of continuous curves, Duke Math. J. 21 (1954), 363-367. MR 15, 977.

4. - Continuous collections of continuous curves in the plane, Proc. Amer. Math. Soc. 3 (1952), 647-657. MR 14, 783.

5. - A continuous curve admitting monotone open maps onto all locally connected metric continua, Bull. Amer. Math. Soc. 62 (1956), 264-265.

6. - Monotone interior dimension-raising mappings, Duke Math. J. 19 (1952), 359-366. MR 14, 71.

7. - One-dimensional continuous curves, Proc. Nat. Acad. Sci. U.S.A. 42 (1956), 760-762. MR 18, 325.

8. - One-dimensional continuous curves and a homogeneity theorem, Ann. of Math.(2), 68 (1958), 1-16. MR 20 \#2676.

9. - Open mappings of compact continua, Proc. Nat. Acad. Sci. U.S.A. 42 (1956), 347-349. MR 17, 1230.

10. - Open mappings of continua, Summer Institute on Set Theoretic Topology, Amer. Math. Soc., Providence, R. I., 1958.

11. R. H. Bing, Partitioning a set, Bull. Amer. Math. Soc. 55 (1949), 1101-1110. MR 11, 733.

12. — , Partitioning continuous curves, Bull. Amer. Math. Soc. 58 (1952), 536-556. MR 14, 192.

13. E. Dyer, Certain transformations which lower dimension, Ann. of Math. (2) 63 (1956), 15-19. MR 17, 993.

14. E. Dyer and M. E. Hamstrom, Completely regular mappings, Fund. Math. 45 (1958), 103-118. MR 19, 1187.

15. L. Keldyš, Example of a one-dimensional continuum with a zero-dimensional and interior mapping onto the square, Dokl. Akad. Nauk SSSR 97 (1954), 201-204. (Russian) MR 16, 60.

16. A. Kolmogoroff, Über offene Abbildungen, Ann. of Math. (2) 38 (1937), 36-38.

17. L. F. McAuley, Open mappings and open problems, Topology Conference (Arizona State University, 1967), Arizona State Univ., Tempe, Ariz., 1968, pp. 184-202. MR 39 \#2134.

18. G. T. Whyburn, Analytic topology, Amer. Math. Soc. Colloq. Publ., vol. 28, Amer. Math. Soc., Providence, R. I., 1963. MR 32 \#425.

Department of Mathematics, Rutgers University, New Brunswick, New Jersey 08903

School of Mathematics, Institute for Advanced Study, Princeton, New Jersey 08540

Current address: Department of Mathematics, Northern Illinois University, DeKalb, ILLINOIS 60115 